\title{
Bicomplex Quantum Mechanics: II. The Hilbert Space
}

\author{
D. Rochon* S. Tremblay ${ }^{\dagger}$ \\ Département de mathématiques et d'informatique \\ Université du Québec à Trois-Rivières \\ C.P. 500 Trois-Rivières, Québec \\ Canada, G9A 5H7
}

\begin{abstract}
Using the bicomplex numbers $\mathbb{T} \cong \mathrm{Cl}_{\mathbb{C}}(1,0) \cong \mathrm{Cl}_{\mathbb{C}}(0,1)$ which is a commutative ring with zero divisors defined by $\mathbb{T}=\left\{w_{0}+w_{1} \mathbf{i}_{\mathbf{1}}+w_{2} \mathbf{i}_{\mathbf{2}}+w_{3} \mathbf{j} \mid w_{0}, w_{1}, w_{2}, w_{3} \in \mathbb{R}\right\}$ where $\mathbf{i}_{\mathbf{1}}^{2}=-1, \mathbf{i}_{\mathbf{2}}^{2}=-1, \mathbf{j}^{2}=1$ and $\mathbf{i}_{\mathbf{1}} \mathbf{i}_{\mathbf{2}}=\mathbf{j}=\mathbf{i}_{\mathbf{2}} \mathbf{i}_{\mathbf{1}}$, we construct hyperbolic and bicomplex Hilbert spaces. Linear functionals and dual spaces are considered on these spaces and properties of linear operators are obtained; in particular it is established that the eigenvalues of a bicomplex self-adjoint operator are in the set of hyperbolic numbers.
\end{abstract}

Keywords: Bicomplex Numbers, Hyperbolic Numbers, Complex Clifford Algebras, Generalized Quantum Mechanics, Hilbert Spaces, Free Modules, Linear Functionals, Self-Adjoint Operators.

\footnotetext{
*E-mail: Dominic.Rochon@UQTR.CA

${ }^{\dagger}$ E-mail: Sebastien.Tremblay@UQTR.CA
} 


\section{Introduction}

Many papers have been written on the extension of the formalism of quantum mechanics. These generalizations have been done mainly over quaternions or over the Cayley algebra (octonions), see for instance 1, 2, 3, 4, The reason why people have worked mainly on this algebraic structures to generalize quantum mechanics comes from the fact that there exist only four normed division algebra $[5]$ : real $(\mathbb{R})$, complex $(\mathbb{C})$, quaternions $(\mathbb{H})$ and Cayley algebra $(\mathbb{O})$. Cayley algebra has an important blank since associativity is crucial. Indeed, in [1] it is shown that quantum mechanics cannot be formulated over the Cayley algebra since, in a last two instances, associativity is needed for the existence of Hilbert space. Quantum mechanics over quaternions seems to work better [1, 2, 3, 6]. However, recently some interest have been deployed to study quantum mechanics for associative and commutative algebras beyond the paradigm of algebras without zero divisors [7, 8, 9]. This leads to a wide spectrum of possibilities, among which we have the hyperbolic numbers $\mathbb{D} \cong \mathrm{Cl}_{\mathbb{R}}(0,1)$ (also called duplex numbers) [10, the bicomplex numbers $\mathbb{T} \cong \mathrm{Cl}_{\mathbb{C}}(1,0) \cong \mathrm{Cl}_{\mathbb{C}}(0,1)$ [1] and, more generally, the multicomplex numbers [12, 13].

In recent years, theory of bicomplex numbers and bicomplex functions has found many applications, see for instance [14, 15, 16, 17, 18. Bicomplex numbers is a commutative ring with unity which contains the field of complex numbers and the commutative ring of hyperbolic numbers. Bicomplex (hyperbolic) numbers are unique among the complex (real) Clifford algebras in that they are commutative but not division algebras. In fact, bicomplex numbers generalize (complexify) hyperbolic numbers. Note that Hilbert spaces over hyperbolic numbers that have been studied in [ 8, 9] and [19] are different from the hyperbolic Hilbert space that we consider in this paper.

In Section 2 we give an overview of the fundamental theory of bicomplex analysis necessary for this article. Section 3 is devoted to free modules over the ring of bicomplex numbers (which is not a $C^{*}$-algebra). A fundamental result useful for the rest of the paper is presented: the unique decomposition of any elements of our free module $M$ into two elements of a standard (complex) vector space in terms of the idempotent basis. The Section 4 (and 5) introduces the bicomplex scalar product (the hyperbolic scalar product). In particular, it is shown that one can constructs a metric space from $M$ and our bicomplex scalar product. In Section 6, we define the bicomplex Hilbert space; two examples are given. Section 7 introduces the dual space $M^{*}$ and re-examines the previous Sections in terms of the Dirac notation. Finally, Section 8 concerns linear operators or more specifically adjoint and self-adjoint operators as well as the bicomplex eigenvectors equation. 


\section{Preliminaries}

Bicomplex numbers are defined as [1, 12, 20,

$$
\mathbb{T}:=\left\{z_{1}+z_{2} \mathbf{i}_{\mathbf{2}} \mid z_{1}, z_{2} \in \mathbb{C}\left(\mathbf{i}_{\mathbf{1}}\right)\right\},
$$

where the imaginary units $\mathbf{i}_{\mathbf{1}}, \mathbf{i}_{\mathbf{2}}$ and $\mathbf{j}$ are governed by the rules: $\mathbf{i}_{\mathbf{1}}^{2}=\mathbf{i}_{\mathbf{2}}^{2}=-1$, $\mathbf{j}^{2}=1$ and

$$
\begin{aligned}
\mathbf{i}_{\mathbf{1}} \mathbf{i}_{\mathbf{2}} & =\mathbf{i}_{\mathbf{2}} \mathbf{i}_{\mathbf{1}}=\mathbf{j}, \\
\mathbf{i}_{\mathbf{1}} \mathbf{j} & =\mathbf{j i}_{\mathbf{1}}=-\mathbf{i}_{\mathbf{2}}, \\
\mathbf{i}_{\mathbf{2}} \mathbf{j} & =\mathbf{j} \mathbf{i}_{\mathbf{2}}=-\mathbf{i}_{\mathbf{1}} .
\end{aligned}
$$

Where we define $\mathbb{C}\left(\mathbf{i}_{k}\right):=\left\{x+y \mathbf{i}_{k} \mid \mathbf{i}_{k}^{2}=-1\right.$ and $\left.x, y \in \mathbb{R}\right\}$ for $k=1,2$. Hence it is easy to see that the multiplication of two bicomplex numbers is commutative.

It is also convenient to write the set of bicomplex numbers as

$$
\mathbb{T}:=\left\{w_{0}+w_{1} \mathbf{i}_{\mathbf{1}}+w_{2} \mathbf{i}_{\mathbf{2}}+w_{3} \mathbf{j} \mid w_{0}, w_{1}, w_{2}, w_{3} \in \mathbb{R}\right\}
$$

In particular, in equation (2.1), if we put $z_{1}=x$ and $z_{2}=y \mathbf{i}_{\mathbf{1}}$ with $x, y \in \mathbb{R}$, then we obtain the subalgebra of hyperbolic numbers: $\mathbb{D}=\left\{x+y \mathbf{j} \mid \mathbf{j}^{2}=1, x, y \in \mathbb{R}\right\}$.

Complex conjugation plays an important role both for algebraic and geometric properties of $\mathbb{C}$, as well as in the standard quantum mechanics. For bicomplex numbers, there are three possible conjugations. Let $w \in \mathbb{T}$ and $z_{1}, z_{2} \in \mathbb{C}\left(\mathbf{i}_{\mathbf{1}}\right)$ such that $w=z_{1}+z_{2} \mathbf{i}_{2}$. Then we define the three conjugations as:

$$
\begin{aligned}
& w^{\dagger_{1}}=\left(z_{1}+z_{2} \mathbf{i}_{2}\right)^{\dagger_{1}}:=\bar{z}_{1}+\bar{z}_{2} \mathbf{i}_{2}, \\
& w^{\dagger_{2}}=\left(z_{1}+z_{2} \mathbf{i}_{2}\right)^{\dagger_{2}}:=z_{1}-z_{2} \mathbf{i}_{2}, \\
& w^{\dagger_{3}}=\left(z_{1}+z_{2} \mathbf{i}_{2}\right)^{\dagger_{3}}:=\bar{z}_{1}-\bar{z}_{2} \mathbf{i}_{2},
\end{aligned}
$$

\begin{tabular}{|c|c|c|c|c|}
\hline 0 & $\dagger_{0}$ & $\dagger_{1}$ & $\dagger_{2}$ & $t_{3}$ \\
\hline$\dagger_{0}$ & $t_{0}$ & $t_{1}$ & $\dagger_{2}$ & $t_{3}$ \\
\hline$t_{1}$ & $t_{1}$ & $t_{0}$ & $t_{3}$ & $t_{2}$ \\
\hline$t_{2}$ & $t_{2}$ & $t_{3}$ & $t_{0}$ & $\dagger_{1}$ \\
\hline$\dagger_{3}$ & $\dagger_{3}$ & $\dagger_{2}$ & $\dagger_{1}$ & $\dagger_{0}$ \\
\hline
\end{tabular}

where $\bar{z}_{k}$ is the standard complex conjugate of complex numbers $z_{k} \in \mathbb{C}\left(\mathbf{i}_{\mathbf{1}}\right)$. If we say that the bicomplex number $w=z_{1}+z_{2} \mathbf{i}_{\mathbf{2}}=w_{0}+w_{1} \mathbf{i}_{\mathbf{1}}+w_{2} \mathbf{i}_{\mathbf{2}}+w_{3} \mathbf{j}$ has the "signature" $(++++)$, then the conjugations of type 1,2 or 3 of $w$ have, respectively, the signatures $(+-+-),(++--)$ and $(+--+)$. We can verify easily that the composition of the conjugates gives the four-dimensional abelian Klein group:

where $w^{\dagger_{0}}:=w \forall w \in \mathbb{T}$. 
The three kinds of conjugation all have some of the standard properties of conjugations, such as:

$$
\begin{aligned}
(s+t)^{\dagger_{k}} & =s^{\dagger_{k}}+t^{\dagger_{k}}, \\
\left(s^{\dagger_{k}}\right)^{\dagger_{k}} & =s \\
(s \cdot t)^{\dagger_{k}} & =s^{\dagger_{k}} \cdot t^{\dagger_{k}},
\end{aligned}
$$

for $s, t \in \mathbb{T}$ and $k=0,1,2,3$.

We know that the product of a standard complex number with its conjugate gives the square of the Euclidean metric in $\mathbb{R}^{2}$. The analogs of this, for bicomplex numbers, are the following. Let $z_{1}, z_{2} \in \mathbb{C}\left(\mathbf{i}_{\mathbf{2}}\right)$ and $w=z_{1}+z_{2} \mathbf{i}_{\mathbf{2}} \in \mathbb{T}$, then we have that [11:

$$
\begin{aligned}
|w|_{\mathbf{i}_{1}}^{2} & :=w \cdot w^{\dagger_{2}}=z_{1}^{2}+z_{2}^{2} \in \mathbb{C}\left(\mathbf{i}_{\mathbf{1}}\right), \\
|w|_{\mathbf{i}_{\mathbf{2}}}^{2} & :=w \cdot w^{\dagger_{1}}=\left(\left|z_{1}\right|^{2}-\left|z_{2}\right|^{2}\right)+2 \operatorname{Re}\left(z_{1} \bar{z}_{2}\right) \mathbf{i}_{\mathbf{2}} \in \mathbb{C}\left(\mathbf{i}_{\mathbf{2}}\right), \\
|w|_{\mathbf{j}}^{2} & :=w \cdot w^{\dagger_{3}}=\left(\left|z_{1}\right|^{2}+\left|z_{2}\right|^{2}\right)-2 \operatorname{Im}\left(z_{1} \bar{z}_{2}\right) \mathbf{j} \in \mathbb{D},
\end{aligned}
$$

where the subscript of the square modulus refers to the subalgebra $\mathbb{C}\left(\mathbf{i}_{\mathbf{1}}\right), \mathbb{C}\left(\mathbf{i}_{\mathbf{2}}\right)$ or $\mathbb{D}$ of $\mathbb{T}$ in which $w$ is projected.

Note that for $z_{1}, z_{2} \in \mathbb{C}\left(\mathbf{i}_{\mathbf{1}}\right)$ and $w=z_{1}+z_{2} \mathbf{i}_{\mathbf{2}} \in \mathbb{T}$, we can define the usual (Euclidean in $\mathbb{R}^{4}$ ) norm of $w$ as $|w|=\sqrt{\left|z_{1}\right|^{2}+\left|z_{2}\right|^{2}}=\sqrt{\operatorname{Re}\left(|w|_{\mathbf{j}}^{2}\right)}$.

It is easy to verify that $w \cdot \frac{w^{\dagger_{2}}}{|w|_{\mathbf{i}_{1}}^{2}}=1$. Hence, the inverse of $w$ is given by

$$
w^{-1}=\frac{w^{\dagger_{2}}}{|w|_{\mathbf{i}_{1}}^{2}} .
$$

From this, we find that the set $\mathcal{N C}$ of zero divisors of $\mathbb{T}$, called the null-cone, is given by $\left\{z_{1}+z_{2} \mathbf{i}_{\mathbf{2}} \mid z_{1}^{2}+z_{2}^{2}=0\right\}$, which can be rewritten as

$$
\mathcal{N C}=\left\{z\left(\mathbf{i}_{\mathbf{1}} \pm \mathbf{i}_{\mathbf{2}}\right) \mid z \in \mathbb{C}\left(\mathbf{i}_{\mathbf{1}}\right)\right\} .
$$

Let us now recall the following three real moduli (see [1] and [20]):

1) For $s, t \in \mathbb{T}$, we define the first modulus as $|\cdot|_{\mathbf{1}}:=\left.|| \cdot\right|_{\mathbf{i}_{1}} \mid$. This modulus has the following properties:
a) $|\cdot|_{1}: \mathbb{T} \rightarrow \mathbb{R}$
b) $|s|_{\mathbf{1}} \geq 0$ with $|s|_{\mathbf{1}}=0$ iff $s \in \mathcal{N C}$;
c) $|s \cdot t|_{\mathbf{1}}=|s|_{\mathbf{1}} \cdot|t|_{\mathbf{1}}$. 
From this definition, we can rewrite this real pseudo-modulus in a much practical way as

$$
|w|_{\mathbf{1}}=\left|z_{1}^{2}+z_{2}^{2}\right|^{1 / 2}
$$

or

$$
|w|_{\mathbf{1}}=\sqrt[4]{w w^{\dagger_{1}} w^{\dagger_{2}} w^{\dagger_{3}}}
$$

2) For $s, t \in \mathbb{T}$, we can define formally the second real modulus as $|\cdot|_{\mathbf{2}}:=$ $\left.|| \cdot\right|_{\mathbf{i}_{2}} \mid$. But an easy computation leads to

$$
|w|_{\mathbf{2}}=|w|_{\mathbf{1}}=\left|z_{1}^{2}+z_{2}^{2}\right|^{1 / 2},
$$

meaning that there are no reasons to introduce $|\cdot|_{\mathbf{2}}$.

3) One more option is to define the third modulus as $|\cdot|_{\mathbf{3}}:=|| \cdot\left|{ }_{\mathbf{j}}\right|$. It has the following properties:

a) $|\cdot|_{3}: \mathbb{T} \rightarrow \mathbb{R}$

b) $|s|_{\mathbf{3}} \geq 0$ with $|s|_{\mathbf{3}}=0$ iff $s=0$

c) $|s+t|_{\mathbf{3}} \leq|s|_{\mathbf{3}}+|t|_{\mathbf{3}}$

d) $|s \cdot t|_{\mathbf{3}} \leq \sqrt{2}|s|_{\mathbf{3}} \cdot|t|_{\mathbf{3}}$

e) $|\lambda \cdot t|_{\mathbf{3}}=|\lambda| \cdot|t|_{\mathbf{3}}$, for $\lambda \in \mathbb{C}\left(\mathbf{i}_{\mathbf{1}}\right)$ or $\mathbb{C}\left(\mathbf{i}_{\mathbf{2}}\right)$.

Hence $|\cdot|_{\mathbf{3}}$ determines a structure of a real normed algebra on $\mathbb{T}$. What is more, one gets directly that

$$
|w|_{\mathbf{3}}=\sqrt{\left|z_{1}\right|^{2}+\left|z_{2}\right|^{2}}
$$

for $w=z_{1}+z_{2} \mathbf{i}_{\mathbf{2}}$ with $z_{1}, z_{2} \in \mathbb{C}\left(\mathbf{i}_{\mathbf{1}}\right)$, i.e., in fact this is just the Euclidean metric in $\mathbb{R}^{4}$ written in a form compatible with the multiplicative structure of bicomplex numbers.

Note also that

(i) $|w|_{\mathbf{j}}=\left|z_{1}-z_{2} \mathbf{i}_{\mathbf{1}}\right| \mathbf{e}_{\mathbf{1}}+\left|z_{1}+z_{2} \mathbf{i}_{\mathbf{1}}\right| \mathbf{e}_{\mathbf{2}} \in \mathbb{D}, \quad \forall w=z_{1}+z_{2} \mathbf{i}_{\mathbf{2}} \in \mathbb{T}$,

(ii) $|s \cdot t|_{\mathbf{j}}=|s|_{\mathbf{j}}|t|_{\mathbf{j}} \quad \forall s, t \in \mathbb{T}$.

Finally, let us mention that any bicomplex numbers can be written using an orthogonal idempotent basis defined by

$$
\mathbf{e}_{\mathbf{1}}=\frac{1+\mathbf{j}}{2} \quad \text { and } \quad \mathbf{e}_{2}=\frac{1-\mathbf{j}}{2}
$$

where $\mathbf{e}_{\mathbf{1}}^{2}=\mathbf{e}_{\mathbf{1}}, \mathbf{e}_{\mathbf{2}}^{2}=\mathbf{e}_{\mathbf{2}}, \mathbf{e}_{\mathbf{1}}+\mathbf{e}_{\mathbf{2}}=1$ and $\mathbf{e}_{\mathbf{1}} \mathbf{e}_{\mathbf{2}}=0=\mathbf{e}_{\mathbf{2}} \mathbf{e}_{\mathbf{1}}$. Indeed, it is easy to show that for any $z_{1}+z_{2} \mathbf{i}_{\mathbf{2}} \in \mathbb{T}, z_{1}, z_{2} \in \mathbb{C}\left(\mathbf{i}_{1}\right)$, we have

$$
z_{1}+z_{2} \mathbf{i}_{\mathbf{2}}=\left(z_{1}-z_{2} \mathbf{i}_{\mathbf{1}}\right) \mathbf{e}_{\mathbf{1}}+\left(z_{1}+z_{2} \mathbf{i}_{\mathbf{1}}\right) \mathbf{e}_{\mathbf{2}}
$$




\section{$3 \quad \mathbb{T}$-Module}

The set of bicomplex number is a commutative ring. So, to define a kind of vector space over $\mathbb{T}$, we have to deal with the algebraic concept of module. We denote $M$ as a free $\mathbb{T}$-module with the following finit $\mathbb{T}$-basis $\left\{\widehat{m}_{l} \mid l \in\right.$ $\{1, \ldots, n\}\}$. Hence,

$$
M=\left\{\sum_{l=1}^{n} x_{l} \widehat{m}_{l} \mid x_{l} \in \mathbb{T}\right\} .
$$

Let us now define

$$
V:=\left\{\sum_{l=1}^{n} x_{l} \widehat{m}_{l} \mid x_{l} \in \mathbb{C}\left(\mathbf{i}_{\mathbf{1}}\right)\right\} \subset M .
$$

The set $V$ is a free $\mathbb{C}\left(\mathbf{i}_{1}\right)$-module which depends on a given $\mathbb{T}$-basis of $M$. In fact, $V$ is a complex vector space of dimension $n$ with the basis $\left\{\widehat{m}_{l} \mid l \in\{1, \ldots, n\}\right\}$. For a complete traitement of the Module Theory, see [21].

Theorem 1 Let $\widehat{X}=\sum_{l=1}^{n} x_{l} \widehat{m}_{l}, x_{l} \in \mathbb{T}, \forall l \in\{1, \ldots, n\}$. Then, there exist $\widehat{X}_{\mathbf{e}_{1}}, \widehat{X}_{\mathbf{e}_{2}} \in V$ such that

$$
\widehat{X}=\mathbf{e}_{1} \widehat{X}_{\mathbf{e}_{1}}+\mathbf{e}_{2} \widehat{X}_{\mathbf{e}_{2}}
$$

Proof. From equation (2.14), it is always possible to decompose a bicomplex number in term of the idempotent basis. So let us write $x_{l}=x_{1 l} \mathbf{e}_{\mathbf{1}}+x_{2 l} \mathbf{e}_{\mathbf{2}}$ where $x_{1 l}, x_{2 l} \in \mathbb{C}\left(\mathbf{i}_{1}\right)$, for all $l \in\{1, \ldots, n\}$. Hence,

$$
\begin{aligned}
\widehat{X} & =\sum_{l=1}^{n} x_{l} \widehat{m}_{l}=\sum_{l=1}^{n}\left(x_{1 l} \mathbf{e}_{\mathbf{1}}+x_{2 l} \mathbf{e}_{\mathbf{2}}\right) \widehat{m}_{l}=\mathbf{e}_{\mathbf{1}} \sum_{l=1}^{n}\left(x_{1 l} \widehat{m}_{l}\right)+\mathbf{e}_{\mathbf{2}} \sum_{l=1}^{n}\left(x_{2 l} \widehat{m}_{l}\right) \\
& =\mathbf{e}_{\mathbf{1}} \widehat{X}_{\mathbf{e}_{\mathbf{1}}}+\mathbf{e}_{\mathbf{2}} \widehat{X}_{\mathbf{e}_{\mathbf{2}}}
\end{aligned}
$$

where $\widehat{X}_{\mathbf{e}_{\mathbf{k}}}:=\sum_{l=1}^{n}\left(x_{k l} \widehat{m}_{l}\right)$ for $k=1,2$.

Corollary 1 The elements $\widehat{X}_{\mathbf{e}_{1}}$ and $\widehat{X}_{\mathbf{e}_{2}}$ are uniquely determined. In other words, $\mathbf{e}_{1} \widehat{X}_{\mathbf{e}_{1}}+\mathbf{e}_{2} \widehat{X}_{\mathbf{e}_{2}}=\mathbf{e}_{1} \widehat{Y}_{\mathbf{e}_{1}}+\mathbf{e}_{2} \widehat{Y}_{\mathbf{e}_{2}}$ if and only if $\widehat{X}_{\mathbf{e}_{1}}=\widehat{Y}_{\mathbf{e}_{1}}$ and $\widehat{X}_{\mathbf{e}_{2}}=\widehat{Y}_{\mathbf{e}_{2}}$.

Proof. If $\mathbf{e}_{1} \widehat{X}_{\mathbf{e}_{1}}+\mathbf{e}_{2} \widehat{X}_{\mathbf{e}_{2}}=\mathbf{e}_{1} \widehat{Y}_{\mathbf{e}_{1}}+\mathbf{e}_{2} \widehat{Y}_{\mathbf{e}_{2}}$, then we have $\mathbf{e}_{\mathbf{1}}\left(\widehat{X}_{\mathbf{e}_{1}}-\widehat{Y}_{\mathbf{e}_{1}}\right)+$ $\mathbf{e}_{2}\left(\widehat{X}_{\mathbf{e}_{2}}-\widehat{Y}_{\mathbf{e}_{2}}\right)=\widehat{0}$. Suppose now that $\left\{\widehat{m}_{l} \mid l \in\{1, \ldots, n\}\right\}$ is a free basis of $M$, 
then we have $\widehat{X}_{\mathbf{e}_{\mathbf{k}}}=\sum_{l=1}^{n} x_{k l} \widehat{m}_{l}$ and $\widehat{Y}_{\mathbf{e}_{\mathbf{k}}}=\sum_{l=1}^{n} y_{k l} \widehat{m}_{l}(k=1,2), x_{k l}, y_{k l} \in \mathbb{C}\left(\mathbf{i}_{\mathbf{1}}\right)$. Therefore, we find

$$
\begin{aligned}
\widehat{0} & =\mathbf{e}_{\mathbf{1}}\left(\widehat{X}_{\mathbf{e}_{\mathbf{1}}}-\widehat{Y}_{\mathbf{e}_{\mathbf{1}}}\right)+\mathbf{e}_{\mathbf{2}}\left(\widehat{X}_{\mathbf{e}_{\mathbf{2}}}-\widehat{Y}_{\mathbf{e}_{\mathbf{2}}}\right) \\
& =\mathbf{e}_{\mathbf{1}}\left(\sum_{l=1}^{n} x_{1 l} \widehat{m}_{l}-\sum_{l=1}^{n} y_{1 l} \widehat{m}_{l}\right)+\mathbf{e}_{\mathbf{2}}\left(\sum_{l=1}^{n} x_{2 l} \widehat{m}_{l}-\sum_{l=1}^{n} y_{2 l} \widehat{m}_{l}\right) \\
& =\sum_{l=1}^{n}\left(x_{l}-y_{l}\right) \widehat{m}_{l},
\end{aligned}
$$

where $x_{l}:=\mathbf{e}_{\mathbf{1}} x_{1 l}+\mathbf{e}_{\mathbf{2}} x_{2 l} \in \mathbb{T}$ and $y_{l}:=\mathbf{e}_{1} y_{1 l}+\mathbf{e}_{\mathbf{2}} y_{2 l} \in \mathbb{T}$. This implies that $x_{l}=y_{l}$ for all $l \in\{1, \ldots, n\}$; in other words $x_{1 l}=y_{2 l}$ and $x_{2 l}=y_{2 l}$, i.e. $\widehat{X}_{\mathbf{e}_{\mathbf{k}}}=\widehat{Y}_{\mathbf{e}_{\mathbf{k}}}$ for $k=1,2$.

Conversely, if $\widehat{X}_{\mathbf{e}_{1}}=\widehat{Y}_{\mathbf{e}_{1}}$ and $\widehat{X}_{\mathbf{e}_{2}}=\widehat{Y}_{\mathbf{e}_{2}}$ we find trivially the desired result. Whenever $\widehat{X} \in M$, we define the projection $P_{k}: M \longrightarrow V$ as

$$
P_{k}(\widehat{X}):=\widehat{X}_{\mathbf{e}_{\mathbf{k}}}
$$

for $k=1,2$. This definition is a generalization of the mutually complementary projections $\left\{P_{1}, P_{2}\right\}$ defined in [11] on $\mathbb{T}$, where $\mathbb{T}$ is considered as the canonical $\mathbb{T}$-module over the ring of bicomplex numbers. Moreover, from the Corollary 1 $\widehat{X}_{\mathbf{e}_{1}}$ and $\widehat{X}_{\mathbf{e}_{\mathbf{2}}}$ are uniquely determined from a given $\mathbb{T}$-basis and the projections $P_{1}$ and $P_{2}$ satisfies the following property:

$$
P_{k}\left(w_{1} \widehat{X}+w_{2} \widehat{Y}\right)=P_{k}\left(w_{1}\right) P_{k}(\widehat{X})+P_{k}\left(w_{2}\right) P_{k}(\widehat{Y})
$$

$\forall w_{1}, w_{2} \in \mathbb{T}, \forall \widehat{X}, \widehat{Y} \in M$ and $k=1,2$.

The vector space $V$ is defined from the free $\mathbb{T}$-module $M$ with a given $\mathbb{T}$-basis. The next theorem tell us that $M$ is isomorphic to $V^{2}=\{(\widehat{X} ; \widehat{Y}) \mid \widehat{X}, \widehat{Y} \in V\}$, where the addition $+_{V^{2}}$ and the multiplication $\cdot^{2}$ by a scalar are defined by

$$
\begin{aligned}
& +_{V^{2}}: \quad V^{2} \times V^{2} \rightarrow V^{2} \\
& \left(\left(\widehat{X}_{1} ; \widehat{Y}_{1}\right),\left(\widehat{X}_{2} ; \widehat{Y}_{2}\right)\right) \mapsto\left(\widehat{X}_{1} ; \widehat{Y}_{1}\right)+_{V_{2}}\left(\widehat{X}_{2} ; \widehat{Y}_{2}\right) \\
& :=\left(\widehat{X}_{1}+\widehat{X}_{2} ; \widehat{Y}_{1}+\widehat{Y}_{2}\right), \\
& \cdot V^{2}: \quad \mathbb{T} \times V^{2} \rightarrow V^{2} \\
& (\lambda,(\widehat{X} ; \widehat{Y})) \quad \mapsto \quad \lambda \cdot V^{2}(\widehat{X} ; \widehat{Y}) \\
& :=\left(\lambda_{1} \widehat{X} ; \lambda_{2} \widehat{Y}\right),
\end{aligned}
$$

where $\lambda=\lambda_{1} \mathbf{e}_{1}+\lambda_{2} \mathbf{e}_{2}$. Here the symbol + denotes the addition on $V$ and $\lambda_{1} \widehat{X}$ or $\lambda_{2} \widehat{Y}$ denotes the multiplication by a scalar on $V$ (which are also the addition and the multiplication defined on $M$ ). Note that we use the notation $(\widehat{X} ; \widehat{Y})$ to denote an element of $V^{2}$, instead of the usual notation $(\widehat{X}, \widehat{Y})$, to avoid confusion with the bicomplex scalar product defined below. 
Theorem 2 The set $V^{2}$ defined with the addition $+_{V_{2}}$ and the multiplication by a scalar $\cdot V^{2}$ over the bicomplex numbers $\mathbb{T}$ is isomorphic to $M$, i.e.

$$
\left(V^{2},{ }_{V_{2}}, \cdot V^{2}\right) \simeq(M,+, \cdot) .
$$

Proof. It is first easy to show that $V^{2}$ is a $\mathbb{T}$-module with $+_{V^{2}}$ and $\cdot_{V^{2}}$ defined above. Now let us consider the function $\Phi: V^{2} \rightarrow M$ defined by $\Phi((\widehat{X} ; \widehat{Y}))=\mathbf{e}_{1} \widehat{X}+\mathbf{e}_{\mathbf{2}} \widehat{Y}$. It is not difficult to show that $\Phi\left(\left(\widehat{X}_{1} ; \widehat{Y}_{1}\right)+{ }_{V^{2}}\right.$ $\left.\left(\widehat{X}_{2} ; \widehat{Y}_{2}\right)\right)=\Phi\left(\left(\widehat{X}_{1} ; \widehat{Y}_{1}\right)\right)+\Phi\left(\left(\widehat{X}_{2} ; \widehat{Y}_{2}\right)\right)$ and that $\Phi\left(\lambda \cdot V^{2} \widehat{X}\right)=\lambda \Phi(\widehat{X})$, i.e. that $\Phi$ is an homomorphism. The function $\Phi$ is a one-to-one function. Indeed if $\Phi\left(\left(\widehat{X}_{1} ; \widehat{Y}_{1}\right)\right)=\Phi\left(\left(\widehat{X}_{2} ; \widehat{Y}_{2}\right)\right)$, then $\mathbf{e}_{\mathbf{1}} \widehat{X}_{1}+\mathbf{e}_{2} \widehat{Y}_{1}=\mathbf{e}_{1} \widehat{X}_{2}+\mathbf{e}_{2} \widehat{Y}_{2}$ which implies that $\widehat{X}_{1}=\widehat{X}_{2}$ and $\widehat{Y}_{1}=\widehat{Y}_{2}$ from Corollary 1 Finally, $\Phi$ is an onto function since for all $\widehat{X}=\mathbf{e}_{1} \widehat{X}_{\mathbf{e}_{1}}+\mathbf{e}_{\mathbf{2}} \widehat{X}_{\mathbf{e}_{2}} \in M$, we have $\Phi\left(\left(\widehat{X}_{\mathbf{e}_{1}} ; \widehat{X}_{\mathbf{e}_{2}}\right)\right)=\widehat{X}$.

Theorem 3 Let $\left\{\widehat{v}_{l} \mid l \in\{1, \ldots, n\}\right\}$ a basis of the vector space $V$ over $\mathbb{C}\left(\mathbf{i}_{1}\right)$. Then $\left\{\left(\widehat{v}_{l} ; \widehat{v}_{l}\right) \mid l \in\{1, \ldots, n\}\right\}$ is a basis of the free $\mathbb{T}$-module $\left(V^{2},+_{V^{2}}, \cdot V^{2}\right)$ and $\left\{\widehat{v}_{l} \mid l \in\{1, \ldots, n\}\right\}$ is a $\mathbb{T}$-basis of $M$.

Proof. Let us consider an arbitrary $(\widehat{X} ; \widehat{Y}) \in V^{2}$, then

$$
(\widehat{X} ; \widehat{Y})=\left(\sum_{l=1}^{n} c_{1 l} \widehat{v}_{l} ; \sum_{l=1}^{n} c_{2 l} \widehat{v}_{l}\right)=\sum_{l=1}^{n}\left(c_{1 l} \widehat{v}_{l} ; c_{2 l} \widehat{v}_{l}\right),
$$

with $c_{k l} \in \mathbb{C}\left(\mathbf{i}_{\mathbf{1}}\right)(k=1,2)$. Here the summations in the second expression is the addition on $V$ and the summation in the third expression is the addition over $V^{2}$, i.e. the addition $+_{V^{2}}$. Therefore, we have

$$
(\widehat{X} ; \widehat{Y})=\sum_{l=1}^{n} c_{l} \cdot V^{2}\left(\widehat{v}_{l} ; \widehat{v}_{l}\right),
$$

where $c_{l}=\mathbf{e}_{1} c_{1 l}+\mathbf{e}_{2} c_{2 l} \in \mathbb{T}$. Moreover, if $(\widehat{X} ; \widehat{Y})=(\widehat{0} ; \widehat{0})$, then $c_{1 l}=c_{2 l}=0$ for all $l \in\{1, \ldots, n\}$ since $\left\{\widehat{v}_{l} \mid l \in\{1, \ldots, n\}\right\}$ is a basis of $V$ and $c_{l}=0$ for all $l \in\{1, \ldots, n\}$. Therefore $\left\{\left(\widehat{v}_{l} ; \widehat{v}_{l}\right) \mid l \in\{1, \ldots, n\}\right\}$ is a $\mathbb{T}$-basis of $V^{2}$ and the $\mathbb{T}$-module $\left(V^{2},+_{V^{2}}, \cdot V^{2}\right)$ is free. It is now easy to see that $\left\{\widehat{v}_{l} \mid l \in\{1, \ldots, n\}\right\}$ is a $\mathbb{T}$-basis of $M$ since the isomorphism $\Phi$ given in the proof of Theorem 2 gives $\Phi\left(\left(\widehat{v}_{l} ; \widehat{v}_{l}\right)\right)=\mathbf{e}_{\mathbf{1}} \widehat{v}_{l}+\mathbf{e}_{\mathbf{2}} \widehat{v}_{l}=\widehat{v}_{l}$ for all $l \in\{1, \ldots, n\}$.

Remark. For $(\widehat{X} ; \widehat{Y}) \in V^{2}$, we have

$$
\begin{aligned}
(\widehat{X} ; \widehat{Y}) & =(\widehat{X} ; \widehat{0})+V_{V^{2}}(\widehat{0} ; \widehat{Y}) \\
& =\left(1 \mathbf{e}_{\mathbf{1}}+0 \mathbf{e}_{\mathbf{2}}\right) \cdot V^{2}(\widehat{X} ; \widehat{X})+_{V^{2}}\left(0 \mathbf{e}_{\mathbf{1}}+1 \mathbf{e}_{\mathbf{2}}\right) \cdot V^{2}(\widehat{Y} ; \widehat{Y}) \\
& =\mathbf{e}_{\mathbf{1}} \cdot V^{2}(\widehat{X} ; \widehat{X})+{ }_{V^{2}} \mathbf{e}_{\mathbf{2}} \cdot V^{2}(\widehat{Y} ; \widehat{Y}),
\end{aligned}
$$


where $(\widehat{X} ; \widehat{X})$ and $(\widehat{Y} ; \widehat{Y})$ are in the vector space $V^{\prime}:=\left\{\sum_{l=1}^{n} c_{l} \cdot V^{2}\left(\widehat{v}_{l} ; \widehat{v}_{l}\right) \mid c_{l} \in\right.$ $\left.\mathbb{C}\left(\mathbf{i}_{\mathbf{1}}\right)\right\}$ associated with the free $\mathbb{T}$-module $V^{2}$ using the following $\mathbb{T}$-basis $\left\{\left(\widehat{v}_{l} ; \widehat{v}_{l}\right) \mid\right.$ $l \in\{1, \ldots, n\}\}$.

Now, from Theorem 3 we obtain the following corollary.

Corollary 2 Let $M$ be a free $\mathbb{T}$-module with a finit $\mathbb{T}$-basis. The submodule vector space $V$ associated with $M$ is invariant under a new $\mathbb{T}$-basis of $M$ generated by another basis of $V$.

\section{Bicomplex scalar product}

Let us begin with a preliminary definition.

Definition 1 A hyperbolic number $w=a \mathbf{e}_{\mathbf{1}}+\mathbf{b}_{\mathbf{2}}$ is define to be positive if $a, b \in \mathbb{R}^{+}$. We denote the set of all positive hyperbolic numbers by

$$
\mathbb{D}^{+}:=\left\{a \mathbf{e}_{\mathbf{1}}+b \mathbf{e}_{\mathbf{2}} \mid a, b \geq 0\right\} .
$$

We are now able to give a definition of a bicomplex scalar product. (In this article, the physicist convention will be used for the order of the elements in the bicomplex scalar product.)

Definition 2 Let $M$ be a free $\mathbb{T}$-module of finit dimension. With each pair $\widehat{X}$ and $\widehat{Y}$ in $M$, taken in this order, we associate a bicomplex number, which is their bicomplex scalar product $(\widehat{X}, \widehat{Y})$, and which satisfies the following properties:

1. $\left(\widehat{X}, \widehat{Y}_{1}+\widehat{Y}_{2}\right)=\left(\widehat{X}, \widehat{Y}_{1}\right)+\left(\widehat{X}, \widehat{Y}_{2}\right), \forall \widehat{X}, \widehat{Y}_{1}, \widehat{Y}_{2} \in M$

2. $(\widehat{X}, \alpha \widehat{Y})=\alpha(\widehat{X}, \widehat{Y}), \forall \alpha \in \mathbb{T}, \forall \widehat{X}, \widehat{Y} \in M$;

3. $(\widehat{X}, \widehat{Y})=(\widehat{Y}, \widehat{X})^{\dagger 3}, \forall \widehat{X}, \widehat{Y} \in M$

4. $(\widehat{X}, \widehat{X})=0 \Leftrightarrow \widehat{X}=0, \forall \widehat{X} \in M$.

As a consequence of property 3 , we have that $(\widehat{X}, \widehat{X}) \in \mathbb{D}$. Note that definition 2 is a general definition of a bicomplex scalar product. However, in this article we will also require the bicomplex scalar product $(\cdot, \cdot)$ to be hyperbolic positive, i.e.

$$
(\widehat{X}, \widehat{X}) \in \mathbb{D}^{+}, \forall \widehat{X} \in M
$$

and closed under the vector space $V$, i.e.

$$
(\widehat{X}, \widehat{Y}) \in \mathbb{C}\left(\mathbf{i}_{\mathbf{1}}\right), \forall \widehat{X}, \widehat{Y} \in V
$$

For the rest of this paper, we will assume a given $\mathbb{T}$-basis for $M$, which implies a given vector space $V$. 
Theorem 4 Let $\widehat{X}, \widehat{Y} \in M$, then

$$
(\widehat{X}, \widehat{Y})=\mathbf{e}_{1}\left(\widehat{X}_{\mathbf{e}_{1}}, \widehat{Y}_{\mathbf{e}_{1}}\right)+\mathbf{e}_{\mathbf{2}}\left(\widehat{X}_{\mathbf{e}_{2}}, \widehat{Y}_{\mathbf{e}_{2}}\right)
$$

and

$$
P_{k}((\widehat{X}, \widehat{Y}))=(\widehat{X}, \widehat{Y})_{\mathbf{e}_{\mathbf{k}}}=\left(\widehat{X}_{\mathbf{e}_{\mathbf{k}}}, \widehat{Y}_{\mathbf{e}_{\mathbf{k}}}\right) \in \mathbb{C}\left(\mathbf{i}_{\mathbf{1}}\right)
$$

for $k=1,2$.

Proof. From equation (3.2), it comes automatically that $P_{k}((\widehat{X}, \widehat{Y}))=(\widehat{X}, \widehat{Y})_{\mathbf{e}_{\mathbf{k}}} \in$ $\mathbb{C}\left(\mathbf{i}_{1}\right)$ for $k=1,2$. Let $\widehat{X}=\mathbf{e}_{1} \widehat{X}_{\mathbf{e}_{1}}+\mathbf{e}_{\mathbf{2}} \widehat{X}_{\mathbf{e}_{2}}$ and $\widehat{Y}=\mathbf{e}_{1} \widehat{Y}_{\mathbf{e}_{1}}+\mathbf{e}_{\mathbf{2}} \widehat{Y}_{\mathbf{e}_{2}}$, then using the properties of the bicomplex scalar product, we also have

$$
\begin{aligned}
& (\widehat{X}, \widehat{Y})=\left(\mathbf{e}_{1} \widehat{X}_{\mathbf{e}_{1}}+\mathbf{e}_{2} \widehat{X}_{\mathbf{e}_{2}}, \mathbf{e}_{1} \widehat{Y}_{\mathbf{e}_{1}}+\mathbf{e}_{\mathbf{2}} \widehat{Y}_{\mathbf{e}_{2}}\right) \\
& =\left(\mathbf{e}_{1} \widehat{X}_{\mathbf{e}_{1}}+\mathbf{e}_{2} \widehat{X}_{\mathbf{e}_{2}}, \mathbf{e}_{1} \widehat{Y}_{\mathbf{e}_{1}}\right)+\left(\mathbf{e}_{1} \widehat{X}_{\mathbf{e}_{1}}+\mathbf{e}_{2} \widehat{X}_{\mathbf{e}_{2}}, \mathbf{e}_{\mathbf{2}} \widehat{Y}_{\mathbf{e}_{2}}\right) \\
& =\left(\mathbf{e}_{\mathbf{1}} \widehat{Y}_{\mathbf{e}_{\mathbf{1}}}, \mathbf{e}_{\mathbf{1}} \widehat{X}_{\mathbf{e}_{\mathbf{1}}}+\mathbf{e}_{\mathbf{2}} \widehat{X}_{\mathbf{e}_{\mathbf{2}}}\right)^{\dagger 3}+\left(\mathbf{e}_{\mathbf{2}} \widehat{Y}_{\mathbf{e}_{\mathbf{2}}}, \mathbf{e}_{\mathbf{1}} \widehat{X}_{\mathbf{e}_{\mathbf{1}}}+\mathbf{e}_{\mathbf{2}} \widehat{X}_{\mathbf{e}_{\mathbf{2}}}\right)^{\dagger 3} \\
& =\left(\mathbf{e}_{1} \widehat{Y}_{\mathbf{e}_{1}}, \mathbf{e}_{1} \widehat{X}_{\mathbf{e}_{1}}\right)^{\dagger_{3}}+\left(\mathbf{e}_{\mathbf{1}} \widehat{Y}_{\mathbf{e}_{\mathbf{1}}}, \mathbf{e}_{\mathbf{2}} \widehat{X}_{\mathbf{e}_{\mathbf{2}}}\right)^{\dagger_{3}} \\
& +\left(\mathbf{e}_{\mathbf{2}} \widehat{Y}_{\mathbf{e}_{2}}, \mathbf{e}_{\mathbf{1}} \widehat{X}_{\mathbf{e}_{\mathbf{1}}}\right)^{\dagger_{3}}+\left(\mathbf{e}_{\mathbf{2}} \widehat{Y}_{\mathbf{e}_{2}}, \mathbf{e}_{\mathbf{2}} \widehat{X}_{\mathbf{e}_{2}}\right)^{\dagger_{3}} \\
& =\mathbf{e}_{\mathbf{1}}{ }^{\dagger_{3}}\left(\mathbf{e}_{\mathbf{1}} \widehat{Y}_{\mathbf{e}_{\mathbf{1}}}, \widehat{X}_{\mathbf{e}_{\mathbf{1}}}\right)^{\dagger_{3}}+\mathbf{e}_{\mathbf{2}}{ }^{\dagger_{3}}\left(\mathbf{e}_{\mathbf{1}} \widehat{Y}_{\mathbf{e}_{\mathbf{1}}}, \widehat{X}_{\mathbf{e}_{\mathbf{2}}}\right)^{\dagger_{3}} \\
& +\mathbf{e}_{\mathbf{1}}{ }^{\dagger_{3}}\left(\mathbf{e}_{\mathbf{2}} \widehat{Y}_{\mathbf{e}_{\mathbf{2}}}, \widehat{X}_{\mathbf{e}_{\mathbf{1}}}\right)^{\dagger_{3}}+\mathbf{e}_{\mathbf{2}}{ }^{\dagger_{3}}\left(\mathbf{e}_{\mathbf{2}} \widehat{Y}_{\mathbf{e}_{\mathbf{2}}}, \widehat{X}_{\mathbf{e}_{2}}\right)^{\dagger_{3}} \\
& =\mathbf{e}_{\mathbf{1}}{ }^{\dagger 3} \mathbf{e}_{\mathbf{1}}\left(\widehat{X}_{\mathbf{e}_{1}}, \widehat{Y}_{\mathbf{e}_{\mathbf{1}}}\right)+\mathbf{e}_{\mathbf{2}}{ }^{\dagger 3} \mathbf{e}_{\mathbf{1}}\left(\widehat{X}_{\mathbf{e}_{\mathbf{2}}}, \widehat{Y}_{\mathbf{e}_{\mathbf{1}}}\right) \\
& +\mathbf{e}_{1}{ }^{\dagger_{3}} \mathbf{e}_{\mathbf{2}}\left(\widehat{X}_{\mathbf{e}_{1}}, \widehat{Y}_{\mathbf{e}_{\mathbf{2}}}\right)+\mathbf{e}_{\mathbf{2}}{ }^{\dagger_{3}} \mathbf{e}_{\mathbf{2}}\left(\widehat{X}_{\mathbf{e}_{\mathbf{2}}}, \widehat{Y}_{\mathbf{e}_{\mathbf{2}}}\right) \\
& =\mathbf{e}_{1}\left(\widehat{X}_{\mathbf{e}_{1}}, \widehat{Y}_{\mathbf{e}_{1}}\right)+\mathbf{e}_{2}\left(\widehat{X}_{\mathbf{e}_{2}}, \widehat{Y}_{\mathbf{e}_{2}}\right) \text {. }
\end{aligned}
$$

Hence,

$$
(\widehat{X}, \widehat{Y})=\mathbf{e}_{\mathbf{1}}\left(\widehat{X}_{\mathbf{e}_{1}}, \widehat{Y}_{\mathbf{e}_{1}}\right)+\mathbf{e}_{\mathbf{2}}\left(\widehat{X}_{\mathbf{e}_{2}}, \widehat{Y}_{\mathbf{e}_{2}}\right)
$$

and, from property (4.2), we obtain

$$
P_{k}((\widehat{X}, \widehat{Y}))=(\widehat{X}, \widehat{Y})_{\mathbf{e}_{\mathbf{k}}}=\left(\widehat{X}_{\mathbf{e}_{\mathbf{k}}}, \widehat{Y}_{\mathbf{e}_{\mathbf{k}}}\right) \in \mathbb{C}\left(\mathbf{i}_{\mathbf{1}}\right)
$$

for $k=1,2$.

Theorem $5\{V ;(\cdot, \cdot)\}$ is a complex $\left(\right.$ in $\left.\mathbb{C}\left(\mathbf{i}_{1}\right)\right)$ pre-Hilbert space.

Proof. By definition, $V \subseteq M$. Hence, we obtain automatically that:

1. $\left(\widehat{X}, \widehat{Y}_{1}+\widehat{Y}_{2}\right)=\left(\widehat{X}, \widehat{Y}_{1}\right)+\left(\widehat{X}, \widehat{Y}_{2}\right), \forall \widehat{X}, \widehat{Y}_{1}, \widehat{Y}_{2} \in V$

2. $(\widehat{X}, \alpha \widehat{Y})=\alpha(\widehat{X}, \widehat{Y}), \forall \alpha \in \mathbb{C}\left(\mathbf{i}_{1}\right)$ and $\forall \widehat{X}, \widehat{Y} \in V$;

3. $(\widehat{X}, \widehat{X})=0 \Leftrightarrow \widehat{X}=0, \forall \widehat{X} \in V$.

Moreover, the fact that $(\widehat{X}, \widehat{Y}) \in \mathbb{C}\left(\mathbf{i}_{1}\right)$ implies that $(\widehat{X}, \widehat{Y})=(\widehat{Y}, \widehat{X})^{\dagger_{3}}=\overline{(\widehat{Y}, \widehat{X})}$ and $(\widehat{X}, \widehat{X}) \in \mathbb{D}^{+} \cap \mathbb{C}\left(\mathbf{i}_{\mathbf{1}}\right)=\mathbb{R}^{+}$. Hence, $\{V ;(\cdot, \cdot)\}$ is a complex (in $\left.\mathbb{C}\left(\mathbf{i}_{\mathbf{1}}\right)\right)$ preHilbert space.

Remark. We note that the results obtained in this theorem are still valid by using $\dagger_{1}$ instead of $\dagger_{3}$ in the definition of the bicomplex scalar product.

Let us denote $\|\widehat{X}\|:=(\widehat{X}, \widehat{X})^{\frac{1}{2}}, \forall \widehat{X} \in V$. 
Corollary 3 Let $\widehat{X} \in V$. The function $\widehat{X} \longmapsto\|\widehat{X}\| \geq 0$ is a norm on $V$.

Corollary 4 Let $\widehat{X} \in M$, then

$$
P_{k}((\widehat{X}, \widehat{X}))=(\widehat{X}, \widehat{X})_{\mathbf{e}_{\mathbf{k}}}=\left(\widehat{X}_{\mathbf{e}_{\mathbf{k}}}, \widehat{X}_{\mathbf{e}_{\mathbf{k}}}\right)=\left\|\widehat{X}_{\mathbf{e}_{\mathbf{k}}}\right\|^{2}
$$

for $k=1,2$.

Now, let us extend this norm on $M$ with the following function:

$$
\|\widehat{X}\|:=\left|(\widehat{X}, \widehat{X})^{\frac{1}{2}}\right|=\left|\mathbf{e}_{\mathbf{1}}\left\|\widehat{X}_{\mathbf{e}_{\mathbf{1}}}\right\|+\mathbf{e}_{\mathbf{2}}\left\|\widehat{X}_{\mathbf{e}_{\mathbf{2}}}\right\|\right|, \forall \widehat{X} \in M .
$$

This norm has the following properties.

Theorem 6 Let $\widehat{X}, \widehat{Y} \in M$ and $d(\widehat{X}, \widehat{Y}):=\|\widehat{X}-\widehat{Y}\|$, then

1. $\|\widehat{X}\| \geq 0$

2. $\|\widehat{X}\|=0 \Leftrightarrow \widehat{X}=0$;

3. $\|\alpha \widehat{X}\|=|\alpha|\|\widehat{X}\|, \forall \alpha \in \mathbb{C}\left(\mathbf{i}_{1}\right)$ or $\mathbb{C}\left(\mathbf{i}_{2}\right)$;

4. $\|\alpha \widehat{X}\| \leq \sqrt{2}|\alpha|_{3}\|\widehat{X}\|, \forall \alpha \in \mathbb{T}$;

5. $\|\widehat{X}+\widehat{Y}\| \leq\|\widehat{X}\|+\|\widehat{Y}\|$;

6. $\{M, d\}$ is a metric space.

Proof. The proof of 1 and 2 come directly from equation (4.5). Let $\widehat{X}=$ $\mathbf{e}_{\mathbf{1}} \widehat{X}_{\mathbf{e}_{\mathbf{1}}}+\mathbf{e}_{\mathbf{2}} \widehat{X}_{\mathbf{e}_{\mathbf{2}}} \in M$ and $\alpha \in \mathbb{C}\left(\mathbf{i}_{1}\right)$ or $\mathbb{C}\left(\mathbf{i}_{\mathbf{2}}\right)$, then

$$
\begin{aligned}
\|\alpha \widehat{X}\| & =\left|(\alpha \widehat{X}, \alpha \widehat{X})^{\frac{1}{2}}\right| \\
& =\left|(\alpha \bar{\alpha}(\widehat{X}, \widehat{X}))^{\frac{1}{2}}\right| \\
& =\left|\left(\mathbf{e}_{1}|\alpha|^{2}(\widehat{X}, \widehat{X})_{\mathbf{e}_{1}}+\mathbf{e}_{2}|\alpha|^{2}(\widehat{X}, \widehat{X})_{\mathbf{e}_{2}}\right)^{\frac{1}{2}}\right| \\
& =\left|\mathbf{e}_{1}\right| \alpha\left|(\widehat{X}, \widehat{X})_{\mathbf{e}_{1}}^{\frac{1}{2}}+\mathbf{e}_{2}\right| \alpha\left|(\widehat{X}, \widehat{X})_{\mathbf{e}_{2}}^{\frac{1}{2}}\right| \\
& =|\alpha|\left|\mathbf{e}_{1}\left\|\widehat{X}_{\mathbf{e}_{1}}\right\|+\mathbf{e}_{\mathbf{2}}\left\|\widehat{X}_{\mathbf{e}_{2}}\right\|\right| \\
& =|\alpha|\|\widehat{X}\| .
\end{aligned}
$$


More generally, if $\alpha \in \mathbb{T}$, we obtain

$$
\begin{aligned}
\|\alpha \widehat{X}\| & =\left|(\alpha \widehat{X}, \alpha \widehat{X})^{\frac{1}{2}}\right| \\
& =\left|\left(\alpha \alpha^{\dagger_{3}}(\widehat{X}, \widehat{X})\right)^{\frac{1}{2}}\right| \\
& =\left|\left(|\alpha|_{\mathbf{j}}^{2}(\widehat{X}, \widehat{X})\right)^{\frac{1}{2}}\right| \\
& =\left.|| \alpha\right|_{\mathbf{j}}(\widehat{X}, \widehat{X})^{\frac{1}{2}} \mid \\
& =\left.|| \alpha\right|_{\mathbf{j}}\|\widehat{X}\| \mid \\
& \leq\left.\sqrt{2}|| \alpha\right|_{\mathbf{j}} \mid\|\widehat{X}\| \\
& =\sqrt{2}|\alpha|_{\mathbf{3}}\|\widehat{X}\| .
\end{aligned}
$$

To complete the proof, we need to establish a triangular inequality over the $\mathbb{T}$-module $M$. Let $\widehat{X}, \widehat{Y} \in M$, then

$$
\begin{aligned}
\|\widehat{X}+\widehat{Y}\| & =\left|(\widehat{X}+\widehat{Y}, \widehat{X}+\widehat{Y})^{\frac{1}{2}}\right| \\
& =\left|\mathbf{e}_{\mathbf{1}}\left\|(\widehat{X}+\widehat{Y})_{\mathbf{e}_{1}}\right\|+\mathbf{e}_{\mathbf{2}}\left\|(\widehat{X}+\widehat{Y})_{\mathbf{e}_{2}}\right\|\right| \\
& =\left|\mathbf{e}_{\mathbf{1}}\left\|\widehat{X}_{\mathbf{e}_{1}}+\widehat{Y}_{\mathbf{e}_{1}}\right\|+\mathbf{e}_{\mathbf{2}}\left\|\widehat{X}_{\mathbf{e}_{2}}+\widehat{Y}_{\mathbf{e}_{2}}\right\|\right| \\
& =\left(\frac{\left\|\widehat{X}_{\mathbf{e}_{1}}+\widehat{Y}_{\mathbf{e}_{1}}\right\|^{2}+\left\|\widehat{X}_{\mathbf{e}_{2}}+\widehat{Y}_{\mathbf{e}_{2}}\right\|^{2}}{2}\right)^{\frac{1}{2}} \\
& \leq\left(\frac{\left(\left\|\widehat{X}_{\mathbf{e}_{1}}\right\|+\left\|\widehat{Y}_{\mathbf{e}_{1}}\right\|\right)^{2}+\left(\left\|\widehat{X}_{\mathbf{e}_{2}}\right\|+\left\|\widehat{Y}_{\mathbf{e}_{2}}\right\|\right)^{2}}{2}\right)^{\frac{1}{2}} \\
& =\left|\mathbf{e}_{1}\left(\left\|\widehat{X}_{\mathbf{e}_{1}}\right\|+\left\|\widehat{Y}_{\mathbf{e}_{1}}\right\|\right)+\mathbf{e}_{\mathbf{2}}\left(\left\|\widehat{X}_{\mathbf{e}_{2}}\right\|+\left\|\widehat{Y}_{\mathbf{e}_{2}}\right\|\right)\right| \\
& =\left|\left(\mathbf{e}_{\mathbf{1}}\left\|\widehat{X}_{\mathbf{e}_{1}}\right\|+\mathbf{e}_{\mathbf{2}}\left\|\widehat{X}_{\mathbf{e}_{2}}\right\|\right)+\left(\mathbf{e}_{\mathbf{1}}\left\|\widehat{Y}_{\mathbf{e}_{1}}\right\|+\mathbf{e}_{\mathbf{2}}\left\|\widehat{Y}_{\mathbf{e}_{2}}\right\|\right)\right| \\
& \leq\|\widehat{X}\|+\| \widehat{Y}_{\|} .
\end{aligned}
$$

Now, using properties $1,2,3$ and 5 , it is easy to obtain that $\{M, d\}$ is a metric space.

With the bicomplex scalar product, it is possible to obtain a bicomplex version of the well known Schwarz inequality.

Theorem 7 (Bicomplex Schwarz inequality) Let $\widehat{X}, \widehat{Y} \in M$ then

$$
|(\widehat{X}, \widehat{Y})| \leq\left|(\widehat{X}, \widehat{X})^{\frac{1}{2}}(\widehat{Y}, \widehat{Y})^{\frac{1}{2}}\right| \leq \sqrt{2}\|\widehat{X}\|\|\widehat{Y}\| .
$$

Proof. From the complex (in $\mathbb{C}\left(\mathbf{i}_{1}\right)$ ) Schwarz inequality we have that

$$
|(\widehat{X}, \widehat{Y})| \leq\|\widehat{X}\|\|\widehat{Y}\| \forall \widehat{X}, \widehat{Y} \in V .
$$


Therefore, if $\widehat{X}, \widehat{Y} \in M$, we obtain

$$
\begin{aligned}
|(\widehat{X}, \widehat{Y})| & =\left|\mathbf{e}_{\mathbf{1}}(\widehat{X}, \widehat{Y})_{\mathbf{e}_{1}}+\mathbf{e}_{\mathbf{2}}(\widehat{X}, \widehat{Y})_{\mathbf{e}_{2}}\right| \\
& =\left|\mathbf{e}_{\mathbf{1}}\left(\widehat{X}_{\mathbf{e}_{1}}, \widehat{Y}_{\mathbf{e}_{1}}\right)+\mathbf{e}_{\mathbf{2}}\left(\widehat{X}_{\mathbf{e}_{2}}, \widehat{Y}_{\mathbf{e}_{2}}\right)\right| \\
& =\left(\frac{\left|\left(\widehat{X}_{\mathbf{e}_{1}}, \widehat{Y}_{\mathbf{e}_{\mathbf{1}}}\right)\right|^{2}+\left|\left(\widehat{X}_{\mathbf{e}_{2}}, \widehat{Y}_{\mathbf{e}_{2}}\right)\right|^{2}}{2}\right)^{\frac{1}{2}} \\
& \leq\left(\frac{\left\|\widehat{X}_{\mathbf{e}_{1}}\right\|^{2}\left\|\widehat{Y}_{\mathbf{e}_{1}}\right\|^{2}+\left\|\widehat{X}_{\mathbf{e}_{2}}\right\|^{2}\left\|\widehat{Y}_{\mathbf{e}_{2}}\right\|^{2}}{2}\right)^{\frac{1}{2}} \\
& =\left|\mathbf{e}_{\mathbf{1}}\left\|\widehat{X}_{\mathbf{e}_{1}}\right\|\left\|\widehat{Y}_{\mathbf{e}_{1}}\right\|+\mathbf{e}_{\mathbf{2}}\left\|\widehat{X}_{\mathbf{e}_{2}}\right\|\left\|\widehat{Y}_{\mathbf{e}_{2}}\right\|\right| \\
& =\left|(\widehat{X}, \widehat{X})^{\frac{1}{2}}(\widehat{Y}, \widehat{Y})^{\frac{1}{2}}\right| .
\end{aligned}
$$

Hence, $|(\widehat{X}, \widehat{Y})| \leq\left|(\widehat{X}, \widehat{X})^{\frac{1}{2}}(\widehat{Y}, \widehat{Y})^{\frac{1}{2}}\right| \leq \sqrt{2}\|\widehat{X}\|\|\widehat{Y}\|$.

\section{$5 \quad$ Hyperbolic scalar product}

From the preceding section, it is now easy to define the hyperbolic version of the bicomplex scalar product.

Definition 3 Let $M$ be a free $\mathbb{D}$-module of finit dimension. With each pair $\widehat{X}$ and $\widehat{Y}$ in $M$, taken in this order, we associate a hyperbolic number, which is their hyperbolic scalar product $(\widehat{X}, \widehat{Y})$, and which satisfies the following properties:

1. $\left(\widehat{X}, \widehat{Y}_{1}+\widehat{Y}_{2}\right)=\left(\widehat{X}, \widehat{Y}_{1}\right)+\left(\widehat{X}, \widehat{Y}_{2}\right)$;

2. $(\widehat{X}, \alpha \widehat{Y})=\alpha(\widehat{X}, \widehat{Y}), \forall \alpha \in \mathbb{D}$;

3. $(\widehat{X}, \widehat{Y})=(\widehat{Y}, \widehat{X})$;

4. $(\widehat{X}, \widehat{X})=0 \Leftrightarrow \widehat{X}=0$.

All definitions and results of Section 4 can be applied directly in the hyperbolic case if the hyperbolic scalar product $(\cdot, \cdot)$ is hyperbolic positive i.e.

$$
(\widehat{X}, \widehat{X}) \in \mathbb{D}^{+} \forall \widehat{X} \in M
$$

and closed under the real vector space $V:=\left\{\sum_{l=1}^{n} x_{l} \widehat{m}_{l} \mid x_{l} \in \mathbb{R}\right\}$ i.e.

$$
(\widehat{X}, \widehat{Y}) \in \mathbb{C}\left(\mathbf{i}_{1}\right) \cap \mathbb{D}=\mathbb{R} \quad \forall \widehat{X}, \widehat{Y} \in V
$$

for a specific $\mathbb{D}$-basis $\left\{\widehat{m}_{l} \mid l \in\{1, \ldots, n\}\right\}$ of $M$. In particular, we obtain an hyperbolic Schwarz inequality. Moreover, it is always possible to obtain the 
angle $\theta$, between $\widehat{X}$ and $\widehat{Y}$ in $V$, with the following well known formula:

$$
\cos \theta=\frac{(\widehat{X}, \widehat{Y})}{\|\widehat{X}\|\|\widehat{Y}\|}
$$

From this result, we can derive the following analogue result for the $\mathbb{D}$-module $M$.

Theorem 8 Let $\widehat{X}, \widehat{Y} \in M$ and $\theta_{k}$ the angle between $\widehat{X}_{\mathbf{e}_{\mathbf{k}}}$ and $\widehat{Y}_{\mathbf{e}_{\mathbf{k}}}$ for $k=1,2$. Then,

$$
\cos \left(\frac{\theta_{1}+\theta_{2}}{2}+\frac{\theta_{1}-\theta_{2}}{2} \mathbf{j}\right)=\frac{(\widehat{X}, \widehat{Y})}{(\widehat{X}, \widehat{X})^{\frac{1}{2}}(\widehat{Y}, \widehat{Y})^{\frac{1}{2}}} .
$$

Proof. From the identity (5.3), we have

$$
\begin{aligned}
\left(\cos \theta_{1}\right) \mathbf{e}_{1}+\left(\cos \theta_{2}\right) \mathbf{e}_{2} & =\frac{\left(\widehat{X}_{\mathbf{e}_{1}}, \widehat{Y}_{\mathbf{e}_{1}}\right)}{\left\|\widehat{X}_{\mathbf{e}_{1}}\right\|\left\|\widehat{Y}_{\mathbf{e}_{1}}\right\|} \mathbf{e}_{1}+\frac{\left(\widehat{X}_{\mathbf{e}_{2}}, \widehat{Y}_{\mathbf{e}_{\mathbf{2}}}\right)}{\left\|\widehat{X}_{\mathbf{e}_{2}}\right\|\left\|\widehat{Y}_{\mathbf{e}_{2}}\right\|} \mathbf{e}_{\mathbf{2}} \\
& =\frac{(\widehat{X}, \widehat{Y})}{(\widehat{X}, \widehat{X})^{\frac{1}{2}}(\widehat{Y}, \widehat{Y})^{\frac{1}{2}}} .
\end{aligned}
$$

Moreover, it is easy to show that $\cos \left(\theta_{1} \mathbf{e}_{\mathbf{1}}+\theta_{2} \mathbf{e}_{\mathbf{2}}\right)=\left(\cos \theta_{1}\right) \mathbf{e}_{\mathbf{1}}+\left(\cos \theta_{2}\right) \mathbf{e}_{\mathbf{2}}$ and $\theta_{1} \mathbf{e}_{1}+\theta_{2} \mathbf{e}_{2}=\frac{\theta_{1}+\theta_{2}}{2}+\frac{\theta_{1}-\theta_{2}}{2} \mathbf{j}$ (see [12]). Hence, $\cos \left(\frac{\theta_{1}+\theta_{2}}{2}+\frac{\theta_{1}-\theta_{2}}{2} \mathbf{j}\right)=$ $\frac{(\widehat{X}, \widehat{Y})}{(\widehat{X}, \widehat{X})^{\frac{1}{2}}(\widehat{Y}, \widehat{Y})^{\frac{1}{2}}} \cdot \square$

From this result, it is now possible to define the "hyperbolic angle" between two elements of a $\mathbb{D}$-module $M$.

Definition 4 Let $\widehat{X}, \widehat{Y} \in M$ and $\theta_{k}$ the angle between $\widehat{X}_{\mathbf{e}_{\mathbf{k}}}$ and $\widehat{Y}_{\mathbf{e}_{\mathbf{k}}}$ for $k=1,2$. We define the hyperbolic angle between $\widehat{X}$ and $\widehat{Y}$ as

$$
\frac{\theta_{1}+\theta_{2}}{2}+\frac{\theta_{1}-\theta_{2}}{2} \mathbf{j}
$$

We note that our definition of the hyperbolic scalar product is different from the definitions given in [8, 9] and [19].

\section{Bicomplex Hilbert space}

Definition 5 Let $M$ be a free $\mathbb{T}$-module with a finit $\mathbb{T}$-basis. Let also $(\cdot, \cdot)$ be a bicomplex scalar product defined on $M$. The space $\{M,(\cdot, \cdot)\}$ is called a $\mathbb{T}$-inner product space.

Definition 6 A complete $\mathbb{T}$-inner product space is called a $\mathbb{T}$-Hilbert space. 
Lemma 1 Let $\widehat{X} \in M$ then

$$
\left\|\widehat{X}_{\mathbf{e}_{\mathbf{k}}}\right\| \leq \sqrt{2}\|\widehat{X}\|, \text { for } k=1,2 .
$$

Proof. For $k=1,2$, we have

$$
\begin{aligned}
\left\|\widehat{X}_{\mathbf{e}_{\mathbf{k}}}\right\| & \leq \sqrt{2}\left(\frac{\left\|\widehat{X}_{\mathrm{e}_{1}}\right\|^{2}+\left\|\widehat{X}_{\mathrm{e}_{2}}\right\|^{2}}{2}\right)^{\frac{1}{2}} \\
& =\sqrt{2}\left|\mathbf{e}_{1}\left\|\widehat{X}_{\mathbf{e}_{1}}\right\|+\mathbf{e}_{\mathbf{2}}\left\|\widehat{X}_{\mathbf{e}_{2}}\right\|\right| \\
& =\sqrt{2}\|\widehat{X}\| .
\end{aligned}
$$

Lemma 2 The pre-Hilbert space $\{V,(\cdot, \cdot)\}$ is closed in the metric space $\{M,(\cdot, \cdot)\}$.

Proof. Let $\widehat{X}_{n}=\mathbf{e}_{\mathbf{1}} \widehat{X}_{n}+\mathbf{e}_{\mathbf{2}} \widehat{X}_{n} \in V \forall n \in \mathbb{N}$ and $\widehat{X}=\mathbf{e}_{\mathbf{1}} \widehat{X}_{\mathbf{e}_{\mathbf{1}}}+\mathbf{e}_{\mathbf{2}} \widehat{X}_{\mathbf{e}_{\mathbf{2}}} \in M$. Supposed that $\widehat{X}_{n} \rightarrow \widehat{X}$ whenever $n \rightarrow \infty$ then $\left\|\widehat{X}_{n}-\widehat{X}\right\| \rightarrow 0$ as $n \rightarrow \infty$ i.e. $\left\|\widehat{X}_{n}-\left(\mathbf{e}_{1} \widehat{X}_{\mathbf{e}_{1}}+\mathbf{e}_{2} \widehat{X}_{\mathbf{e}_{2}}\right)\right\|=\left\|\left(\mathbf{e}_{1} \widehat{X}_{n}+\mathbf{e}_{2} \widehat{X}_{n}\right)-\left(\mathbf{e}_{1} \widehat{X}_{\mathbf{e}_{1}}+\mathbf{e}_{\mathbf{2}} \widehat{X}_{\mathbf{e}_{2}}\right)\right\|=\|$ $\mathbf{e}_{\mathbf{1}}\left(\widehat{X}_{n}-\widehat{X}_{\mathbf{e}_{\mathbf{1}}}\right)+\mathbf{e}_{\mathbf{2}}\left(\widehat{X}_{n}-\widehat{X}_{\mathbf{e}_{\mathbf{2}}}\right) \| \rightarrow 0$ as $n \rightarrow \infty$. Therefore, from the Lemma we have that

$$
\left\|\widehat{X}_{n}-\widehat{X}_{\mathbf{e}_{\mathbf{k}}}\right\| \leq \sqrt{2}\left\|\mathbf{e}_{\mathbf{1}}\left(\widehat{X}_{n}-\widehat{X}_{\mathbf{e}_{\mathbf{1}}}\right)+\mathbf{e}_{\mathbf{2}}\left(\widehat{X}_{n}-\widehat{X}_{\mathbf{e}_{\mathbf{2}}}\right)\right\| \rightarrow 0
$$

as $n \rightarrow \infty$ for $k=1,2$. Hence, $\widehat{X}_{\mathbf{e}_{1}}=\widehat{X}_{\mathbf{e}_{\mathbf{2}}}=\widehat{X}$ and $\widehat{X}=\mathbf{e}_{\mathbf{1}} \widehat{X}+\mathbf{e}_{\mathbf{2}} \widehat{X} \in V$.

Theorem $9 A \mathbb{T}$-inner product space $\{M,(\cdot, \cdot)\}$ is a $\mathbb{T}$-Hilbert space if and only if $\{V,(\cdot, \cdot)\}$ is an Hilbert space.

Proof. From the Theorem $5\{V,(\cdot, \cdot)\}$ is a pre-Hilbert space. So, we have to prove that $\{M,(\cdot, \cdot)\}$ is complete if and only if $\{V,(\cdot, \cdot)\}$ is complete. By definition $V \subseteq M$, therefore if $M$ is complete then $V$ is also complete since $V$ is closed in $M$. Conversely, let $\widehat{X}_{n}=\mathbf{e}_{1}\left(\widehat{X}_{n}\right)_{\mathbf{e}_{1}}+\mathbf{e}_{2}\left(\widehat{X}_{n}\right)_{\mathbf{e}_{2}} \in M \forall n \in \mathbb{N}$, be a Cauchy sequence in $M$. Then, from the Lemma 1 we have

$$
\left\|\left(\widehat{X}_{m}\right)_{\mathbf{e}_{\mathbf{k}}}-\left(\widehat{X}_{n}\right)_{\mathbf{e}_{\mathbf{k}}}\right\|=\left\|\left(\widehat{X}_{m}-\widehat{X}_{n}\right)_{\mathbf{e}_{\mathbf{k}}}\right\| \leq \sqrt{2}\left\|\widehat{X}_{m}-\widehat{X}_{n}\right\|
$$

for $k=1,2$. So, $\left(\widehat{X}_{n}\right)_{\mathbf{e}_{\mathbf{k}}}$ is also a Cauchy sequence in $V$ for $k=1,2$. Therefore, there exist $\widehat{X}_{\mathbf{e}_{1}}, \widehat{X}_{\mathbf{e}_{2}} \in V$ such that $\left(\widehat{X}_{n}\right)_{\mathbf{e}_{\mathbf{k}}} \rightarrow \widehat{X}_{\mathbf{e}_{\mathbf{k}}}$ as $n \rightarrow \infty$ for $k=1,2$.

Now, from the triangular inequality, if we let $\widehat{X}:=\mathbf{e}_{1} \widehat{X}_{\mathbf{e}_{1}}+\mathbf{e}_{2} \widehat{X}_{\mathbf{e}_{2}}$, then we obtain

$$
\begin{aligned}
\left\|\widehat{X}_{n}-\widehat{X}\right\|= & \left\|\mathbf{e}_{\mathbf{1}}\left(\left(\widehat{X}_{n}\right)_{\mathbf{e}_{1}}-\widehat{X}_{\mathbf{e}_{1}}\right)+\mathbf{e}_{\mathbf{2}}\left(\left(\widehat{X}_{n}\right)_{\mathbf{e}_{2}}-\widehat{X}_{\mathbf{e}_{\mathbf{2}}}\right)\right\| \\
\leq & \left\|\mathbf{e}_{\mathbf{1}}\left(\left(\widehat{X}_{n}\right)_{\mathbf{e}_{1}}-\widehat{X}_{\mathbf{e}_{\mathbf{1}}}\right)\right\|+\left\|\mathbf{e}_{\mathbf{2}}\left(\left(\widehat{X}_{n}\right)_{\mathbf{e}_{\mathbf{2}}}-\widehat{X}_{\mathbf{e}_{2}}\right)\right\| \\
\leq & \sqrt{2}\left|\mathbf{e}_{\mathbf{1}}\right|_{\mathbf{3}}\left\|\left(\widehat{X}_{n}\right)_{\mathbf{e}_{1}}-\widehat{X}_{\mathbf{e}_{1}}\right\| \\
& +\sqrt{2}\left|\mathbf{e}_{\mathbf{2}}\right|_{\mathbf{3}}\left\|\left(\widehat{X}_{n}\right)_{\mathbf{e}_{2}}-\widehat{X}_{\mathbf{e}_{2}}\right\| \\
= & \left\|\left(\widehat{X}_{n}\right)_{\mathbf{e}_{\mathbf{1}}}-\widehat{X}_{\mathbf{e}_{\mathbf{1}}}\right\|+\left\|\left(\widehat{X}_{n}\right)_{\mathbf{e}_{\mathbf{2}}}-\widehat{X}_{\mathbf{e}_{\mathbf{2}}}\right\| \rightarrow 0
\end{aligned}
$$

as $n \rightarrow \infty$. Hence, $\widehat{X}_{n} \rightarrow \widehat{X} \in M$ as $n \rightarrow \infty$. 


\section{Examples of bicomplex Hilbert spaces}

1. Let us first consider $M=\mathbb{T}$, the canonical $\mathbb{T}$-module over the ring of bicomplex numbers. We consider now the trivial $\mathbb{T}$-basis $\{1\}$. In this case, the submodule vector space $V$ is simply $V=\mathbb{C}\left(\mathbf{i}_{1}\right)$. Let $(\cdot, \cdot)_{1}$ and $(\cdot, \cdot)_{2}$ be two scalar product on $V$. It is always possible to construct a general bicomplex scalar product as follows:

Let

$$
w_{1}=\left(z_{11}-z_{12} \mathbf{i}_{\mathbf{1}}\right) \mathbf{e}_{\mathbf{1}}+\left(z_{11}+z_{12} \mathbf{i}_{\mathbf{1}}\right) \mathbf{e}_{\mathbf{2}}
$$

and

$$
w_{2}=\left(z_{21}-z_{22} \mathbf{i}_{\mathbf{1}}\right) \mathbf{e}_{\mathbf{1}}+\left(z_{21}+z_{22} \mathbf{i}_{\mathbf{1}}\right) \mathbf{e}_{\mathbf{2}}
$$

where, $z_{11}, z_{12}, z_{21}, z_{22} \in \mathbb{C}\left(\mathbf{i}_{1}\right)$. We define

$$
\left(w_{1}, w_{2}\right):=\left(z_{11}-z_{12} \mathbf{i}_{\mathbf{1}}, z_{21}-z_{22} \mathbf{i}_{\mathbf{1}}\right)_{1} \mathbf{e}_{\mathbf{1}}+\left(z_{11}+z_{12} \mathbf{i}_{\mathbf{1}}, z_{21}+z_{22} \mathbf{i}_{\mathbf{1}}\right)_{2} \mathbf{e}_{\mathbf{2}}
$$

However, this bicomplex scalar product is not closed under $\mathbb{C}\left(\mathbf{i}_{\mathbf{1}}\right)$. In fact, $(\cdot, \cdot)$ will be closed under $\mathbb{C}\left(\mathbf{i}_{\mathbf{1}}\right)$ if and only if $(\cdot, \cdot)_{1}=(\cdot, \cdot)_{2}$. From the Theorem 9] we obtain the following result.

Theorem 10 Let $\mathbb{T}$, the canonical $\mathbb{T}$-module over the ring of bicomplex numbers with a scalar product $(\cdot, \cdot)$ on $\mathbb{C}\left(\mathbf{i}_{\mathbf{1}}\right)$. Let also $w_{1}=\left(z_{11}-\right.$ $\left.z_{12} \mathbf{i}_{\mathbf{1}}\right) \mathbf{e}_{\mathbf{1}}+\left(z_{11}+z_{12} \mathbf{i}_{\mathbf{1}}\right) \mathbf{e}_{\mathbf{2}}$ and $w_{2}=\left(z_{21}-z_{22} \mathbf{i}_{\mathbf{1}}\right) \mathbf{e}_{\mathbf{1}}+\left(z_{21}+z_{22} \mathbf{i}_{\mathbf{1}}\right) \mathbf{e}_{\mathbf{2}}$ where, $z_{11}, z_{12}, z_{21}, z_{22} \in \mathbb{C}\left(\mathbf{i}_{\mathbf{1}}\right)$. If we define

$$
\left(w_{1}, w_{2}\right):=\left(z_{11}-z_{12} \mathbf{i}_{\mathbf{1}}, z_{21}-z_{22} \mathbf{i}_{\mathbf{1}}\right) \mathbf{e}_{\mathbf{1}}+\left(z_{11}+z_{12} \mathbf{i}_{\mathbf{1}}, z_{21}+z_{22} \mathbf{i}_{\mathbf{1}}\right) \mathbf{e}_{\mathbf{2}}
$$

then $\{\mathbb{T},(\cdot, \cdot)\}$ is a bicomplex Hilbert space if and only if $\left\{\mathbb{C}\left(\mathbf{i}_{\mathbf{1}}\right),(\cdot, \cdot)\right\}$ is an Hilbert space.

As an example, let us consider $\left\{\mathbb{C}\left(\mathbf{i}_{1}\right),(\cdot, \cdot)\right\}$ with the canonical scalar product given by

$$
\begin{aligned}
\left(z_{1}, z_{2}\right) & =\left(x_{1}+y_{1} \mathbf{i}_{\mathbf{1}}, x_{2}+y_{2} \mathbf{i}_{\mathbf{1}}\right) \\
& :=x_{1} x_{2}+y_{1} y_{2} .
\end{aligned}
$$

It is well known that $\left\{\mathbb{C}\left(\mathbf{i}_{\mathbf{1}}\right),(\cdot, \cdot)\right\}$ is an Hilbert space. Hence, from the Theorem [10. $\{\mathbb{T},(\cdot, \cdot)\}$ is a bicomplex Hilbert space. Moreover, it is easy to see that

$$
\|w\|=\left.\left.|| w\right|_{\mathbf{j}}|=| w\right|_{\mathbf{3}}=|w|
$$

i.e. the Euclidean metric of $\mathbb{R}^{4}$. 
2. Consider now $M=\mathbb{T}^{n}$, the $n$-dimensional module with the canonical $\mathbb{T}$ basis $\left\{\widehat{e}_{i} \mid i \in\{1, \ldots, n\}\right\}$, the columns of the identity matrix $I_{n}$. For any two elements $\widehat{X}, \widehat{Y} \in \mathbb{T}^{n}$ given by $\widehat{X}=\sum_{i=1}^{n} x_{i} \widehat{e}_{i}$ and $\widehat{Y}=\sum_{i=1}^{n} y_{i} \widehat{e}_{i}$, we define the bicomplex scalar product as

$$
(\widehat{X}, \widehat{Y}):=\left(\widehat{X}^{\dagger_{3}}\right)^{\top} \cdot \widehat{Y}=\sum_{i=1}^{n} x_{i}^{\dagger_{3}} y_{i} \in \mathbb{T} .
$$

It is now easy to verify that properties 1,2 and 3 of Definition 2 are trivially satisfied. This bicomplex scalar product also implies that $(\widehat{X}, \widehat{X})=$ $\sum_{i=1}^{n} x_{i}^{\dagger_{3}} x_{i}=\sum_{i=1}^{n}\left|x_{i}\right|_{\mathbf{j}}^{2}=\mathbf{e}_{\mathbf{1}} \sum_{i=1}^{n}\left|x_{1 i}-x_{2 i} \mathbf{i}_{\mathbf{1}}\right|^{2}+\mathbf{e}_{\mathbf{2}} \sum_{i=1}^{n}\left|x_{1 i}+x_{2 i} \mathbf{i}_{\mathbf{1}}\right|^{2}$ where $x_{i}=x_{1 i}+x_{2 i} \mathbf{i}_{\mathbf{2}}=\left(x_{1 i}-x_{2 i} \mathbf{i}_{\mathbf{1}}\right) \mathbf{e}_{\mathbf{1}}+\left(x_{1 i}+x_{2 i} \mathbf{i}_{\mathbf{1}}\right) \mathbf{e}_{\mathbf{2}}$ for $i \in\{1, \ldots, n\}$. Hence, the property 4 of Definition 2 is also satisfied and

$$
\|\widehat{X}\|=\left|(\widehat{X}, \widehat{X})^{\frac{1}{2}}\right|=\left|\left(\sum_{i=1}^{n}\left|x_{i}\right|_{\mathbf{j}}^{2}\right)^{\frac{1}{2}}\right| .
$$

In this example, the complex vector space $V=\left\{\sum_{i=1}^{n} x_{i} \widehat{e}_{i} \mid x_{i} \in \mathbb{C}\left(\mathbf{i}_{1}\right)\right\}$ is simply the standard complex vector space isomorphic to $\mathbb{C}^{n}$. Moreover, the closure property is satisfied since for $\widehat{X}, \widehat{Y} \in V$ we have $x_{i}, y_{i} \in \mathbb{C}\left(\mathbf{i}_{1}\right)$ and $x_{i}^{\dagger_{3}} y_{i}=\bar{x}_{i} y_{i} \in \mathbb{C}\left(\mathbf{i}_{1}\right)$ such that equation (6.3) gives an element of $\mathbb{C}\left(\mathbf{i}_{1}\right)$.

\section{The Dirac notation over $M$}

In this section we introduce the Dirac notation usually used in quantum mechanics. For this we have to define correctly kets and bras over a bicomplex Hilbert space which, we remind, is fundamentally a module.

Let $M$ be a $\mathbb{T}$-module which is free with the following finit $\mathbb{T}$-basis $\left\{\left|m_{l}\right\rangle \mid\right.$ $l \in\{1, \ldots, n\}\}$. Any element of $M$ will be called a ket module or, more simply, a ket.

Let us rewrite the definition of the bicomplex scalar product in term of the ket notation.

Definition 7 Let $M$ be a $\mathbb{T}$-module which is free with the following finit $\mathbb{T}$-basis $\left\{\left|m_{l}\right\rangle \mid l \in\{1, \ldots, n\}\right\}$. With each pair $|\phi\rangle$ and $|\psi\rangle$ in $M$, taken in this order, we associate a bicomplex number, which is their bicomplex scalar product $(|\phi\rangle,|\psi\rangle)$, and which satisfies the following properties:

1. $\left(|\phi\rangle,\left|\psi_{1}\right\rangle+\left|\psi_{2}\right\rangle\right)=\left(|\phi\rangle,\left|\psi_{1}\right\rangle\right)+\left(|\phi\rangle,\left|\psi_{2}\right\rangle\right)$;

2. $(|\phi\rangle, \alpha|\psi\rangle)=\alpha(|\phi\rangle,|\psi\rangle), \forall \alpha \in \mathbb{T}$;

3. $(|\phi\rangle,|\psi\rangle)=(|\psi\rangle,|\phi\rangle)^{\dagger_{3}}$;

4. $(|\phi\rangle,|\phi\rangle)=0 \Leftrightarrow|\phi\rangle=0$.

Let us now define the dual space $M^{*}$. 
Definition 8 A linear functional $\chi$ is a linear operation which associates a bicomplex number with every ket $|\psi\rangle$ :

1) $|\psi\rangle \longrightarrow \chi(|\psi\rangle) \in \mathbb{T}$;

2) $\chi\left(\lambda_{1}\left|\psi_{1}\right\rangle+\lambda_{2}\left|\psi_{2}\right\rangle\right)=\lambda_{1} \chi\left(\left|\psi_{1}\right\rangle\right)+\lambda_{2} \chi\left(\left|\psi_{2}\right\rangle\right), \quad \lambda_{1}, \lambda_{2} \in \mathbb{T}$.

It can be shown that the set of linear functionals defined on the kets $|\psi\rangle \in M$ constitutes a $\mathbb{T}$-module space, which is called the dual space of $M$ and which will be symbolized by $M^{*}$.

Using this definition of $M^{*}$, let us define the bra notation.

Definition 9 Any element of the space $M^{*}$ is called a bra module or, more simply, a bra. It is symbolized by $\langle\cdot|$.

For example, the bra $\langle\chi|$ designates the bicomplex linear functional $\chi$ and we shall henceforth use the notation $\langle\chi \mid \psi\rangle$ to denote the number obtained by causing the linear functional $\langle\chi| \in M^{*}$ to act on the ket $|\psi\rangle \in M$ :

$$
\chi(|\psi\rangle):=\langle\chi \mid \psi\rangle .
$$

The existence of a bicomplex scalar product in $M$ will now enable us to show that we can associate, with every ket $|\phi\rangle \in M$, an element of $M^{*}$, which will be denoted by $\langle\phi|$.

The ket $|\phi\rangle$ does indeed enable us to define a linear functional: the one which associates (in a linear way), with each ket $|\psi\rangle \in M$, a bicomplex numbers which is equal to the scalar product $(|\phi\rangle,|\psi\rangle)$ of $|\psi\rangle$ by $|\phi\rangle$. Let $\langle\phi|$ be this linear functional; It is thus defined by the relation:

$$
\langle\phi \mid \psi\rangle=(|\phi\rangle,|\psi\rangle) .
$$

Therefore, the properties of the bicomplex scalar product can be rewrited as:

1. $\left\langle\phi\left|\left(\left|\psi_{1}\right\rangle+\left|\psi_{2}\right\rangle\right)=\left\langle\phi \mid \psi_{1}\right\rangle+\left\langle\phi \mid \psi_{2}\right\rangle\right.\right.$;

2. $\langle\phi \mid \alpha \psi\rangle=\alpha\langle\phi \mid \psi\rangle, \forall \alpha \in \mathbb{T}$

3. $\langle\phi \mid \psi\rangle=\langle\psi \mid \phi\rangle^{\dagger 3} ;$

4. $\langle\phi \mid \phi\rangle=0 \Leftrightarrow|\phi\rangle=0$.

Now, let define the corresponding projections for the Dirac notation as follows.

Definition 10 Let $|\psi\rangle,|\phi\rangle \in M$ and $|\chi\rangle \in V$. For $k=1,2$, we define:

1. $\left|\psi_{\mathbf{e}_{\mathbf{k}}}\right\rangle:=P_{k}(|\psi\rangle) \in V$

2. $\left\langle\phi_{\mathbf{e}_{\mathbf{k}}}\right|:=P_{k}(\langle\phi|): V \longrightarrow \mathbb{C}\left(\mathbf{i}_{1}\right)$, where $|\chi\rangle \mapsto P_{k}(\langle\phi \mid \chi\rangle)$.

The first definition gives the projection $\left|\psi_{\mathbf{e}_{\mathbf{k}}}\right\rangle$ of the ket $|\psi\rangle$ of $M$. This is well defined from equation (3.2). However, the second definition is more subtle. In the next two theorems, we show that $\left\langle\phi_{\mathbf{e}_{\mathbf{k}}}\right|$ is really the bra associated with the ket $\left|\phi_{\mathbf{e}_{\mathbf{k}}}\right\rangle$ in $V$. 
Theorem 11 Let $|\phi\rangle \in M$, then

$$
\left\langle\phi_{\mathbf{e}_{\mathbf{k}}}\right| \in V^{*}
$$

for $k=1,2$.

Proof. Let $\lambda_{1}, \lambda_{2} \in \mathbb{C}\left(\mathbf{i}_{1}\right)$ and $\left|\psi_{1}\right\rangle,\left|\psi_{2}\right\rangle \in V$, then

$$
\begin{aligned}
\left\langle\phi _ { \mathbf { e } _ { \mathbf { k } } } \left|\left(\lambda_{1}\left|\psi_{1}\right\rangle+\lambda_{2}\left|\psi_{2}\right\rangle\right)\right.\right. & =P_{k}\left(\left\langle\phi\left|\left(\lambda_{1}\left|\psi_{1}\right\rangle+\lambda_{2}\left|\psi_{2}\right\rangle\right)\right)\right.\right. \\
& =P_{k}\left(\lambda_{1}\left\langle\phi \mid \psi_{1}\right\rangle+\lambda_{2}\left\langle\phi \mid \psi_{2}\right\rangle\right) \\
& =\lambda_{1} P_{k}\left(\left\langle\phi \mid \psi_{1}\right\rangle\right)+\lambda_{2} P_{k}\left(\left\langle\phi \mid \psi_{2}\right\rangle\right) \\
& =\lambda_{1}\left\langle\phi_{\mathbf{e}_{\mathbf{k}}}\right|\left(\left|\psi_{1}\right\rangle\right)+\lambda_{2}\left\langle\phi_{\mathbf{e}_{\mathbf{k}}}\right|\left(\left|\psi_{2}\right\rangle\right)
\end{aligned}
$$

for $k=1,2$.

We will now show that the functional $\left\langle\phi_{\mathbf{e}_{\mathbf{k}}}\right|$ can be obtained from the ket $\left|\phi_{\mathbf{e}_{\mathbf{k}}}\right\rangle$.

Theorem 12 Let $|\phi\rangle \in M$ and $|\psi\rangle \in V$, then

$$
\left\langle\phi_{\mathbf{e}_{\mathbf{k}}}\right|(|\psi\rangle)=\left\langle\phi_{\mathbf{e}_{\mathbf{k}}} \mid \psi\right\rangle
$$

for $k=1,2$.

Proof. Using (4.4) in Theorem 4 and the fact that $P_{k}(|\psi\rangle)=|\psi\rangle$, we obtain

$$
\begin{aligned}
\left\langle\phi_{\mathbf{e}_{\mathbf{k}}}\right|(|\psi\rangle) & =P_{k}(\langle\phi \mid \psi\rangle) \\
& =P_{k}((|\phi\rangle,|\psi\rangle)) \\
& =\left(P_{k}(|\phi\rangle), P_{k}(|\psi\rangle)\right) \\
& =\left(P_{k}(|\phi\rangle),|\psi\rangle\right) \\
& =\left(\left|\phi_{\mathbf{e}_{\mathbf{k}}}\right\rangle,|\psi\rangle\right) \\
& =\left\langle\phi_{\mathbf{e}_{\mathbf{k}}} \mid \psi\right\rangle
\end{aligned}
$$

for $k=1,2$.

Corollary 5 Let $|\phi\rangle,|\psi\rangle \in M$ then

$$
\left\langle\phi_{\mathbf{e}_{\mathbf{k}}} \mid \psi_{\mathbf{e}_{\mathbf{k}}}\right\rangle=\langle\phi \mid \psi\rangle_{\mathbf{e}_{\mathbf{k}}}
$$

for $k=1,2$. 
Proof. From Theorem 12 and the properties of the projectors $P_{k}$, we obtain

$$
\begin{aligned}
\left\langle\phi_{\mathbf{e}_{\mathbf{k}}} \mid \psi_{\mathbf{e}_{\mathbf{k}}}\right\rangle & =P_{k}\left(\left\langle\phi \mid \psi_{\mathbf{e}_{\mathbf{k}}}\right\rangle\right) \\
& =P_{k}\left(\mathbf{e}_{\mathbf{1}}\left\langle\phi \mid \psi_{\mathbf{e}_{1}}\right\rangle+\mathbf{e}_{\mathbf{2}}\left\langle\phi \mid \psi_{\mathbf{e}_{\mathbf{2}}}\right\rangle\right) \\
& =P_{k}\left(\left\langle\phi\left|\left(\mathbf{e}_{1}\left|\psi_{\mathbf{e}_{\mathbf{1}}}\right\rangle+\mathbf{e}_{\mathbf{2}}\left|\psi_{\mathbf{e}_{\mathbf{2}}}\right\rangle\right)\right)\right.\right. \\
& =P_{k}(\langle\phi \mid \psi\rangle) \\
& =\langle\phi \mid \psi\rangle_{\mathbf{e}_{\mathbf{k}}}
\end{aligned}
$$

for $k=1,2$.

The bicomplex scalar product is antilinear. Indeed, by using the notation (7.1) we obtain

$$
\begin{aligned}
\left(\lambda_{1}\left|\phi_{1}\right\rangle+\lambda_{2}\left|\phi_{2}\right\rangle,|\psi\rangle\right) & =\left(|\psi\rangle, \lambda_{1}\left|\phi_{1}\right\rangle+\lambda_{2}\left|\phi_{2}\right\rangle\right)^{\dagger_{3}} \\
& =\left(\lambda_{1}\left\langle\psi \mid \phi_{1}\right\rangle+\lambda_{2}\left\langle\psi \mid \phi_{2}\right\rangle\right)^{\dagger_{3}} \\
& =\lambda_{1}^{\dagger_{3}}\left\langle\phi_{1} \mid \psi\right\rangle+\lambda_{2}^{\dagger_{3}}\left\langle\phi_{2} \mid \psi\right\rangle \\
& =\left(\lambda_{1}^{\dagger_{3}}\left\langle\phi_{1}\left|+\lambda_{2}^{\dagger_{3}}\left\langle\phi_{2}\right|\right) \mid \psi\right\rangle,\right.
\end{aligned}
$$

where $\lambda_{1}, \lambda_{2} \in \mathbb{T}$ and $|\psi\rangle,\left|\phi_{1}\right\rangle,\left|\phi_{2}\right\rangle \in M$. Therefore the bra associated with the ket $\lambda_{1}\left|\phi_{1}\right\rangle+\lambda_{2}\left|\phi_{2}\right\rangle$ is given by $\lambda_{1}^{\dagger_{3}}\left\langle\phi_{1}\right|+\lambda_{2}^{\dagger_{3}}\left\langle\phi_{2}\right|$ :

$$
\lambda_{1}\left|\phi_{1}\right\rangle+\lambda_{2}\left|\phi_{2}\right\rangle \leadsto \lambda_{1}^{\dagger_{3}}\left\langle\phi_{1}\right|+\lambda_{2}^{\dagger_{3}}\left\langle\phi_{2}\right| .
$$

In particular, Theorem 1 tell us that every ket $|\psi\rangle \in M$ can be written in the form $|\psi\rangle=\mathbf{e}_{\mathbf{1}}\left|\psi_{\mathbf{e}_{1}}\right\rangle+\mathbf{e}_{\mathbf{2}}\left|\psi_{\mathbf{e}_{2}}\right\rangle$. Therefore, we have $|\psi\rangle=\mathbf{e}_{1}\left|\psi_{\mathbf{e}_{1}}\right\rangle+\mathbf{e}_{\mathbf{2}}\left|\psi_{\mathbf{e}_{2}}\right\rangle$ um $\langle\psi|=\mathbf{e}_{\mathbf{1}}\left\langle\psi_{\mathbf{e}_{1}}\right|+\mathbf{e}_{\mathbf{2}}\left\langle\psi_{\mathbf{e}_{2}}\right|$ since $\left(\mathbf{e}_{\mathbf{k}}\right)^{\dagger_{3}}=\mathbf{e}_{\mathbf{k}}$ for $k=1,2$.

\section{Bicomplex linear operators}

\subsection{Basic results and definitions}

The bicomplex linear operators $A: M \rightarrow M$ are defined by

$$
\begin{aligned}
& \left|\psi^{\prime}\right\rangle=A|\psi\rangle, \\
& A\left(\lambda_{1}\left|\psi_{1}\right\rangle+\lambda_{2}\left|\psi_{2}\right\rangle\right)=\lambda_{1} A\left|\psi_{1}\right\rangle+\lambda_{2} A\left|\psi_{2}\right\rangle,
\end{aligned}
$$

where $\lambda_{1}, \lambda_{2} \in \mathbb{T}$. For a fixed $|\phi\rangle \in M$, a fixed linear operator $A$ and an arbitrary $|\psi\rangle \in M$, we define the bra $\langle\phi| A$ by the relation

$$
(\langle\phi| A)|\psi\rangle:=\langle\phi|(A|\psi\rangle) .
$$

The operator $A$ associates a new bra $\langle\phi| A$ for every bra $\langle\phi|$. It is easy to show that this correspondance is linear, i.e. $\left(\lambda_{1}\left\langle\phi_{1}\right|+\lambda_{2}\left\langle\phi_{2}\right|\right) A=\lambda_{1}\left\langle\phi_{1}\right| A+\lambda_{2}\left\langle\phi_{2}\right| A$. 
For a given linear operator $A: M \rightarrow M$, the bicomplex adjoint operator $A^{*}$ is the operator with the following correspondance

$$
\left|\psi^{\prime}\right\rangle=A|\psi\rangle \leadsto\left\langle\psi^{\prime}\right|=\langle\psi| A^{*} .
$$

The bicomplex adjoint operator $A^{*}$ is linear: the proof is analogous to the standard case except that the standard complex conjugate is replaced by $\dagger_{3}$ everywhere. Note that since we have $\left\langle\psi^{\prime} \mid \phi\right\rangle=\left\langle\phi \mid \psi^{\prime}\right\rangle^{\dagger_{3}}$, we obtain

$$
\left\langle\psi\left|A^{*}\right| \phi\right\rangle=\langle\phi|A| \psi\rangle^{\dagger}{ }^{3},
$$

by using expressions (8.1).

It is easy to show that for any bicomplex linear operator $A: M \rightarrow M$ and $\lambda \in \mathbb{T}$, we have the following standard properties:

$$
\begin{aligned}
\left(A^{*}\right)^{*} & =A \\
(\lambda A)^{*} & =\lambda^{\dagger_{3}} A^{*}, \\
(A+B)^{*} & =A^{*}+B^{*}, \\
(A B)^{*} & =B^{*} A^{*} .
\end{aligned}
$$

These properties are prove similarly as the standard cases.

Definition 11 Let $M$ be a bicomplex Hilbert space and $A: M \rightarrow M$ a bicomplex linear operator. We define the projection $P_{k}(A): M \rightarrow V$ of $A$, for $k=1,2$, as follows :

$$
P_{k}(A)|\psi\rangle:=P_{k}(A|\psi\rangle), \quad \forall|\psi\rangle \in M .
$$

The projection $P_{k}(A)$ is clearly a bicomplex linear operator for $k=1,2$. Moreover, we have the following specific results.

Theorem 13 Let $M$ be a bicomplex Hilbert space, $A: M \rightarrow M$ a bicomplex linear operator and $|\psi\rangle=\mathbf{e}_{\mathbf{1}}\left|\psi_{\mathbf{e}_{1}}\right\rangle+\mathbf{e}_{\mathbf{2}}\left|\psi_{\mathbf{e}_{2}}\right\rangle \in M$. Then

(i) $A|\psi\rangle=\mathbf{e}_{\mathbf{1}} P_{1}(A)\left|\psi_{\mathbf{e}_{1}}\right\rangle+\mathbf{e}_{\mathbf{2}} P_{2}(A)\left|\psi_{\mathbf{e}_{2}}\right\rangle$;

(ii) $P_{k}(A)^{*}=P_{k}\left(A^{*}\right)$ where $P_{k}(A)^{*}$ is the standard complex adjoint operator over $\mathbb{C}\left(\mathbf{i}_{1}\right)$ associated with the bicomplex linear operator $P_{k}(A)$ restricted to the submodule vector space $V$, defined in (3.1), for $k=1,2$.

Proof. The part $(i)$ is obtain as follows:

$$
\begin{aligned}
A|\psi\rangle= & A\left(\mathbf{e}_{1}\left|\psi_{\mathbf{e}_{1}}\right\rangle+\mathbf{e}_{2}\left|\psi_{\mathbf{e}_{2}}\right\rangle\right) \\
= & \mathbf{e}_{1} A\left|\psi_{\mathbf{e}_{1}}\right\rangle+\mathbf{e}_{2} A\left|\psi_{\mathbf{e}_{2}}\right\rangle \\
= & \mathbf{e}_{1}\left(\mathbf{e}_{1} P_{1}\left(A\left|\psi_{\mathbf{e}_{1}}\right\rangle\right)+\mathbf{e}_{2} P_{2}\left(A\left|\psi_{\mathbf{e}_{1}}\right\rangle\right)\right) \\
& +\mathbf{e}_{2}\left(\mathbf{e}_{1} P_{1}\left(A\left|\psi_{\mathbf{e}_{2}}\right\rangle\right)+\mathbf{e}_{2} P_{2}\left(A\left|\psi_{\mathbf{e}_{2}}\right\rangle\right)\right) \\
= & \mathbf{e}_{1}\left(\mathbf{e}_{1} P_{1}(A)\left|\psi_{\mathbf{e}_{1}}\right\rangle+\mathbf{e}_{2} P_{2}(A)\left|\psi_{\mathbf{e}_{1}}\right\rangle\right) \\
& +\mathbf{e}_{2}\left(\mathbf{e}_{1} P_{1}(A)\left|\psi_{\mathbf{e}_{2}}\right\rangle+\mathbf{e}_{2} P_{2}(A)\left|\psi_{\mathbf{e}_{2}}\right\rangle\right) \\
= & \mathbf{e}_{1} P_{1}(A)\left|\psi_{\mathbf{e}_{1}}\right\rangle+\mathbf{e}_{2} P_{2}(A)\left|\psi_{\mathbf{e}_{2}}\right\rangle .
\end{aligned}
$$


To show (ii), we use (i) and Corollary 5 to decompose the correspondance (8.1) into the equivalent following correspondance in $V$ :

$$
\left|\psi_{\mathbf{e}_{\mathbf{k}}}^{\prime}\right\rangle=P_{k}(A)\left|\psi_{\mathbf{e}_{\mathbf{k}}}\right\rangle \leadsto\left\langle\psi_{\mathbf{e}_{\mathbf{k}}}^{\prime}\right|=\left\langle\psi_{\mathbf{e}_{\mathbf{k}}}\right| P_{k}\left(A^{*}\right) \text { for } k=1,2 .
$$

Hence, $P_{k}(A)^{*}=P_{k}\left(A^{*}\right)$. $\square$

\subsection{Bicomplex eigenvectors and eigenvalues on $M$}

One can show now that the bicomplex eigenvector equation $A|\psi\rangle=\lambda|\psi\rangle$, with $\lambda \in \mathbb{T}$, is equivalent to the system of two eigenvector equations given by

$$
\begin{aligned}
& P_{1}(A)\left|\psi_{\mathbf{e}_{1}}\right\rangle=\lambda_{1}\left|\psi_{\mathbf{e}_{1}}\right\rangle, \\
& P_{2}(A)\left|\psi_{\mathbf{e}_{2}}\right\rangle=\lambda_{2}\left|\psi_{\mathbf{e}_{2}}\right\rangle,
\end{aligned}
$$

where $\lambda=\mathbf{e}_{1} \lambda_{1}+\mathbf{e}_{2} \lambda_{2}, \lambda_{1}, \lambda_{2} \in \mathbb{C}\left(\mathbf{i}_{1}\right)$ and $|\psi\rangle=\mathbf{e}_{\mathbf{1}}\left|\psi_{\mathbf{e}_{1}}\right\rangle+\mathbf{e}_{\mathbf{2}}\left|\psi_{\mathbf{e}_{\mathbf{2}}}\right\rangle$. Indeed, we have

$$
\begin{aligned}
A|\psi\rangle=\lambda|\psi\rangle & \Leftrightarrow A|\psi\rangle=\left(\lambda_{1} \mathbf{e}_{\mathbf{1}}+\lambda_{2} \mathbf{e}_{\mathbf{2}}\right)\left(\mathbf{e}_{\mathbf{1}}\left|\psi_{\mathbf{e}_{1}}\right\rangle+\mathbf{e}_{\mathbf{2}}\left|\psi_{\mathbf{e}_{\mathbf{2}}}\right\rangle\right) \\
& \Leftrightarrow \quad \mathbf{e}_{\mathbf{1}} P_{1}(A)\left|\psi_{\mathbf{e}_{\mathbf{1}}}\right\rangle+\mathbf{e}_{\mathbf{2}} P_{2}(A)\left|\psi_{\mathbf{e}_{\mathbf{2}}}\right\rangle=\mathbf{e}_{\mathbf{1}} \lambda_{1}\left|\psi_{\mathbf{e}_{1}}\right\rangle+\mathbf{e}_{\mathbf{2}} \lambda_{2}\left|\psi_{\mathbf{e}_{\mathbf{2}}}\right\rangle \\
& \Leftrightarrow \quad P_{k}(A)\left|\psi_{\mathbf{e}_{\mathbf{k}}}\right\rangle=\lambda_{k}\left|\psi_{\mathbf{e}_{\mathbf{k}}}\right\rangle, \quad k=1,2 .
\end{aligned}
$$

Suppose now that $\left\{\left|v_{l}\right\rangle \mid l \in\{1, \ldots, n\}\right\}$ is an orthonormal basis of $V$ (which is also a basis of $M$ from Theorem 3) with $\left|\psi_{\mathbf{e}_{\mathbf{k}}}\right\rangle=\sum_{j=1}^{n} c_{k j}\left|v_{j}\right\rangle, c_{k j} \in \mathbb{C}\left(\mathbf{i}_{1}\right)$, $k=1,2$. Then from we find $\sum_{j=1}^{n} c_{k j} P_{k}(A)\left|v_{j}\right\rangle=\lambda_{k} \sum_{j=1}^{n} c_{k j}\left|v_{j}\right\rangle$ for $k=1,2$. Applying now the functional $\left\langle v_{i}\right|$ on this expression, we obtain

$$
\begin{aligned}
\sum_{j=1}^{n} c_{k j}\left\langle v_{i}\left|P_{k}(A)\right| v_{j}\right\rangle & =\lambda_{k} \sum_{j=1}^{n} c_{k j}\left\langle v_{i} \mid v_{j}\right\rangle \\
& =\lambda_{k} c_{k i},
\end{aligned}
$$

where the last line is a consequence of the orthogonality $\left\langle v_{i} \mid v_{j}\right\rangle=\delta_{i j}$ of the basis of $V$. Now, by definition, we have that $P_{k}(A)\left|v_{j}\right\rangle \in V$ for $k=1,2$. Moreover since $\left|v_{i}\right\rangle$ is also an element of $V$ then the closure of the scalar product of two elements of $V$, see equation (4.2), implies that the matrix $A_{k}$ defined by

$$
\left(A_{k}\right)_{i j}:=\left\langle v_{i}\left|P_{k}(A)\right| v_{j}\right\rangle
$$

is in $\mathbb{C}\left(\mathbf{i}_{\mathbf{1}}\right)$ for $k=1,2$. Therefore, we find that

$$
\sum_{j=1}^{n}\left(\left(A_{k}\right)_{i j}-\lambda_{k} \delta_{i j}\right) c_{k j}=0, \quad k=1,2 .
$$


Each equation, i.e. $k=1$ and $k=2$, is a homogeneous linear system with $n$ equations and $n$ unknowns which can be solved completely since all components are in $\mathbb{C}\left(\mathbf{i}_{\mathbf{1}}\right)$. Therefore, the system posses a nontrivial solution if and only $\operatorname{det}\left(A_{k}-\lambda_{k} I_{n}\right)=0$ for $k=1,2$.

In standard quantum mechanics self-adjoint operators (Hermitian operators) play a very important role. In analogy with the standard case, a linear operator $A$ is defined to be a bicomplex self-adjoint operator if and only if $A=A^{*}$.

Theorem 14 Let $A: M \rightarrow M$ be a bicomplex self-adjoint operator and $|\psi\rangle \in M$ be an eigenvector of the equation $A|\psi\rangle=\lambda|\psi\rangle$, with $|\psi\rangle \notin \mathcal{N C}$. Then the eigenvalues of $A$ are in the set of hyperbolic numbers.

Proof. If $A$ is a bicomplex self-adjoint operator $A=A^{*}$ on $M$ and $A|\psi\rangle=\lambda|\psi\rangle$ with $\lambda \in \mathbb{T}$ then

$$
\langle\psi|A| \psi\rangle=\lambda\langle\psi \mid \psi\rangle,
$$

where $\langle\psi \mid \psi\rangle \in \mathbb{D}^{+}$. Moreover, we have

$$
\langle\psi|A| \psi\rangle^{\dagger_{3}}=\left\langle\psi\left|A^{*}\right| \psi\right\rangle=\langle\psi|A| \psi\rangle .
$$

This implies that $\langle\psi|A| \psi\rangle \in \mathbb{D}$. Since $\langle\psi \mid \psi\rangle \notin \mathcal{N C} \Leftrightarrow|\psi\rangle \notin \mathcal{N C}$, we can divide each side of equation (8.9) by $\langle\psi \mid \psi\rangle$. Therefore, $\lambda$ can only be in $\mathbb{D}$.

Remark. The requirement that the eigenvector $|\psi\rangle$ is not in the null-cone means that $|\psi\rangle=\mathbf{e}_{\mathbf{1}}\left|\psi_{\mathbf{e}_{1}}\right\rangle+\mathbf{e}_{\mathbf{2}}\left|\psi_{\mathbf{e}_{2}}\right\rangle$ with $\left|\psi_{\mathbf{e}_{1}}\right\rangle \neq|0\rangle$ and $\left|\psi_{\mathbf{e}_{2}}\right\rangle \neq|0\rangle$. 


\section{References}

[1] S.L. Adler, Quaternionic Quantum Mechanics and Quantum Fields, Oxford University Press, New York (1995).

[2] F.G. Finkelstein et al., Foundations of quaternion quantum mechanics, J. Math Phys. 3, 207-220 (1962).

[3] G. Emch, Mécanique quantique quaternionienne et relativité restreinte. I and II, Helv. Phys. Acta 36, 770-788 (1963).

[4] L.P. Horwitz, Hypercomplex quantum mechanics, Found. Phys. 26, No. 6, 851-862 (1996).

[5] A. Hurwitz, Ueber die Composition der quadratischen Formen von beliebig vielen Variabeln, Nachr. Königl. Gesell. Wiss. Göttingen. Math.Phys. Klasse, 309-316 (1898).

[6] S. De Leo and G.C. Ducati, Quaternionic bound states, J. Phys. A: Math. and Gen. 38, 3443-3454 (2005).

[7] J. Kocik, Duplex numbers, diffusion systems and generalized quantum mechanics, Internat. J. Theor. Phys. 38, No. 8, 2221-2230 (1999).

[8] A. Khrennikov, Ensemble fluctuations and the origin of quantum probabilistic rule, J. Math. Phys. 2, Vol. 43, 789-802 (2002).

[9] A. Khrennikov, Representation of the contextual statistical model by hyperbolic amplitudes, J. Math. Phys. 46, No. 6 (2005).

[10] G. Sobczyk, The hyperbolic number plane, Coll. Maths. Jour. 26, No. 4, 268-280 (1995).

[11] D. Rochon and M. Shapiro, On algebraic properties of bicomplex and hyperbolic numbers, Anal. Univ. Oradea, fasc. math., vol. 11, 71-110 (2004).

[12] G.B. Price, An introduction to multicomplex spaces and functions, Marcel Dekker Inc., New York (1991).

[13] N. Fleury, M. Rausch de Traubenberg and R.M. Yamaleev, Commutative extended complex numbers and connected trigonometry, J. Math. Ann. and Appl. 180, 431-457 (1993).

[14] H. Toyoshima, Computationnally efficient bicomplex multipliers for digital signal processing, IEICE Trans. Inf. \& Syst. E, 80-D, 236-238 (1998).

[15] I.V. Biktasheva and V.N. Biktashev, Response functions of spiral wave solutions of the complex Ginzburg-Landau equation, J. Nonlin. Math. Phys. 8, 28-34 (2001). 
[16] A. Castaneda and V.V. Kravchenko, New applications of pseudoanalytic function theory to the Dirac equation, J. Phys. A.: Math. Gen. 38, 92079219 (2005).

[17] D. Rochon, A generalized Mandelbrot set for bicomplex numbers, Fractal 8, 355-368 (2000).

[18] D. Rochon (2004), A bicomplex Riemann zeta function, Tokyo J. Math. 27, 357-369 (2004).

[19] Y. Xuegang, Hyperbolic Hilbert Space, Adv. App. Cliff. Alg. 10, No. 1, 49-60 (2000).

[20] D. Rochon and S. Tremblay, Bicomplex Quantum Mechanics: I. The Generalized Schrödinger Equation, Adv. App. Cliff. Alg. 12, No. 2, 231-248 (2004).

[21] N. Bourbaki, Éléments de Mathématique VI, Hermann, Paris (1962).

[22] C. Cohen-Tannoudji, B. Diu, and F. Laloë, Mécanique quantique, Hermann, Paris (1977). 